\title{
Fluctuation conductivity of disordered superconductors in magnetic fields
}

\author{
Brian Tarasinski ${ }^{1,2, *}$ and Georg Schwiete ${ }^{1}$ \\ ${ }^{1}$ Dahlem Center for Complex Quantum Systems and Institut für Theoretische Physik, Freie Universität Berlin, 14195 Berlin, Germany \\ ${ }^{2}$ Instituut-Lorentz, Universiteit Leiden, P.O. Box 9506, 2300 RA Leiden, The Netherlands
}

(Received 3 April 2013; revised manuscript received 24 June 2013; published 22 July 2013)

\begin{abstract}
We calculate fluctuation corrections to the longitudinal conductivity of disordered superconductors subject to an external magnetic field. We derive analytic expressions that are valid in the entire metallic part of the temperature-magnetic field phase diagram as long as the effect of the magnetic field on the spin degrees of freedom of the electrons may be neglected. Our calculations are based on a kinetic equation approach. For the special case of superconducting films and wires in parallel magnetic fields, we perform a detailed comparison with results that were previously obtained with diagrammatic perturbation theory in the imaginary-time formalism. As an application, we study the fluctuation conductivity of films in tilted magnetic fields with a special focus on the low-temperature regime. We present a detailed discussion of the phenomenon of the nonmonotonic magnetoresistance and find that it displays a pronounced dependence on the tilting angle.
\end{abstract}

DOI: 10.1103/PhysRevB.88.014518

PACS number(s): 74.40.-n, 74.25.fc

\section{INTRODUCTION}

The theory of superconducting fluctuations has been the subject of intense study for many years. ${ }^{1}$ In the metallic part of the phase diagram, outside the superconducting regime, Cooper pairs may form for a finite time. The presence of these fluctuating Cooper pairs affects both thermodynamic and transport properties of the metal. The phase transition between the metallic and the superconducting state may be tuned by temperature or by so-called pair-breaking mechanisms which lead to a partial or even complete destruction of superconductivity. ${ }^{2}$ Examples of pair breakers include magnetic impurities in $s$-wave superconductors, external magnetic fields, or a flux penetrating a superconductor with doubly connected geometry. Fluctuation effects are particularly strong for low-dimensional superconductors and further enhanced by the presence of impurities. ${ }^{1}$ Detailed experimental ${ }^{3-10}$ and theoretical studies ${ }^{11-22}$ of fluctuation phenomena in superconductors have become available in recent years.

The subject of this paper is the calculation of fluctuation corrections to conductivity in the metallic phase of disordered superconductors. The origin of this field dates back to the work of Azlamazov and Larkin. ${ }^{23}$ These authors studied the direct contribution of fluctuating Cooper pairs to conductivity close to the transition temperature $T_{c 0}$, the so-called paraconductivity. Shortly afterwards, additional contributions were discovered. ${ }^{24,25}$ This development went hand in hand with the study of different classes of diagrams in many-body perturbation theory. It became customary to divide the set of most relevant diagrams into three classes: the AslamazovLarkin diagram and the density of states and Maki-Thompson diagrams. ${ }^{1}$

Initially, studies focused around the vicinity of $T_{c 0}$ for vanishing or small magnetic fields. In Ref. 11, the fluctuation conductivity was calculated for disordered superconducting films in perpendicular magnetic fields in the vicinity of the critical magnetic field $B_{c 2}$. It was established that at very low temperatures, superconducting fluctuations lead to a nonmonotonic magnetoresistance (NM); close to $B_{c 2}$, the resistance curve displays a maximum as a function of the magnetic field. In another theoretical study (Ref. 12), it was found that the NM also exists in the vicinity of certain other classes of pair-breaking transitions such as for films and wires in parallel magnetic fields.

Recently, a novel scheme for deriving fluctuation conductivity was introduced, ${ }^{21}$ which is based on the Usadel equation. ${ }^{26}$ The calculation is performed in the Keldysh formalism to circumvent the analytic continuation necessary in the Kubo technique. ${ }^{27}$ For temperatures close to $T_{c 0}$ and in the absence of a magnetic field, the Usadel equation has been used for the calculation of fluctuation conductivity before. ${ }^{28}$ In Ref. 21, in turn, general expressions for the fluctuation conductivity in disordered superconducting films with perpendicular magnetic field were derived for the whole normal part of the temperature-magnetic field phase diagram (outside the strong fluctuation regime close to the transition line). In this approach, it was possible to identify three distinct contributions to conductivity at the very early stages of the calculation. The first one, termed density of states correction $\left(\delta \sigma_{\text {DOS }}\right)$, is seen to be directly related to the change in the quasiparticle density of states. The second contribution is the anomalous Maki-Thompson correction $\left(\delta \sigma_{\mathrm{an}}\right)$, which is known from diagrammatic perturbation theory and describes a coherent rescattering in the Cooper channel. The third term may be interpreted as the direct contribution of Cooper pairs to the current, and was therefore named supercurrent correction $\left(\delta \sigma_{\mathrm{sc}}\right)$. It should be noted that the density of states and supercurrent contributions in the Usadel equation approach are in general not identical to the contributions of the density of states and Aslamazov-Larkin diagrams in the conventional classification.

Let us briefly recall the origin of the NM for the perpendicular magnetic field case using the language introduced in Ref. 21. We discuss the low-temperature regime $t=T / T_{c 0} \ll$ 1 close to the (temperature-dependent) critical field $B_{c 2}(T)$, so that $h=\left[B-B_{c 2}(T)\right] / B_{c 2}(T) \ll 1$. For $t \gg h$, all corrections, $\delta \sigma_{\mathrm{DOS}}, \delta \sigma_{\mathrm{an}}$, and $\delta \sigma_{\mathrm{sc}}$, contribute, and the total correction to conductivity is positive. As one moves further away from the transition line at fixed temperature, in the limit $t \ll h$, the anomalous Maki-Thompson correction becomes ineffective. The density of states correction to conductivity, which is negative, and the supercurrent correction, which is positive, 
are of similar magnitude. The density of states correction dominates, however, leading to a net negative correction to conductivity. For large magnetic fields, the negative density of states correction is still dominant, but eventually diminishes. The result is a nonmonotonic magnetoresistance. Close to $B_{c 2}$, the results of Ref. 21 coincide with those obtained by Galitski and Larkin, ${ }^{11}$ who specifically focused on this regime and used the conventional diagrammatic method for the calculation. It should be noted that the low-temperature regime is quite different from the well-studied case of small magnetic fields for $T \approx T_{c 0}$. In the latter regime, $\delta \sigma_{\mathrm{sc}}$ is much larger in magnitude than $\delta \sigma_{\text {DOS }}$. The main difference is that close to $B_{c 2}$ Landau-level quantization of the Cooper pair propagator becomes crucial. This is why the supercurrent correction becomes less singular and $\delta \sigma_{\mathrm{sc}}$ and $\delta \sigma_{\mathrm{DOS}}$ are of a comparable magnitude.

In this paper, we use the Usadel equation approach to derive general expressions for the fluctuation conductivity in superconductors subject to a magnetic field. We assume that the sample geometry is translationally invariant along the direction of the electric field, while the sample may be confined in the transverse direction(s). The derived formulas are in particular applicable for superconducting wires, superconducting films in magnetic fields of arbitrary orientation, and for cylinders threaded by a magnetic flux. As a specific application, we study in detail the phenomenon of the NM for films in tilted magnetic fields. We focus on the low-temperature regime, and describe the evolution of the NM as a function of the tilting angle. While the phenomenon persists for any angle, there are two distinct regions: one comprising the parallel magnetic field case and the other one the perpendicular magnetic field case, for which the physical origin of the phenomenon as well as the magnitude of the resulting resistance maximum are quite distinct. The crossover between the two regimes occurs for almost parallel magnetic fields.

The theory developed in this paper is applicable in the limit of weak disorder $\epsilon_{F} \tau \gg 1$, where $\epsilon_{F}$ is the Fermi energy and $\tau$ the transport scattering time. From the experimental perspective, detailed low-temperature resistance measurements have been performed on weakly disordered films in perpendicular magnetic fields (see, e.g., Refs. 29, 30, and 5). Measurements on films in parallel ${ }^{31,32}$ and tilted ${ }^{33}$ magnetic fields exist, but focused on more strongly disordered films in the context of the so-called superconductor-insulator transition.

For the case of superconductors in parallel magnetic fields, we perform a detailed comparison of our results to those obtained in Ref. 12 with the help of the traditional diagrammatic technique. We show that there is a one-to-one correspondence between the results obtained in the two formalisms (up to details of the ultraviolet regularization). The mapping is not simple, however. The three distinct contributions identified in the kinetic equation approach correspond to a mixture of terms originating from different diagrams. This comparison is motivated by a discrepancy between recent results reported for films in perpendicular magnetic fields. Glatz, Varlamov, and Vinokur ${ }^{18,19}$ used the traditional approach in the imaginary-time formalism for the calculation of the fluctuation conductivity. The results of this study disagree with a number of previously reported results, including those for $B \approx B_{c 2}$ (Ref. 11) and the high-temperature regime $T \gg T_{c 0}$ at $B=0$
(Ref. 34). The technically very different Usadel equation approach of Ref. 21, however, confirmed these earlier results. The comparison performed here for the parallel magnetic field case demonstrates an agreement between the imaginary-time formalism as worked out in Ref. 12 and the Usadel equation approach on the level of general formulas valid in the entire normal part of the phase diagram. This includes, in particular, the high-temperature regime $T \gg T_{c 0}$ for $B=0$, which lies within the range of applicability of all the mentioned works.

The paper is organized as follows. In Sec. II, we present the main results of our study. Specifically, in Sec. II A, we display the general formulas for the fluctuation conductivity in superconductors subject to a magnetic field and discuss their range of applicability. In Sec. II B, we discuss the fluctuation conductivity of a thin amorphous superconducting film in a tilted magnetic field. The example of the film in a tilted magnetic field constitutes a special application of the general formalism outlined in this paper. The remainder of the paper is devoted to the technical details of the approach as well as to a comparison with the traditional diagrammatic technique. In Sec. III, we introduce the Usadel equation approach underlying the calculation of fluctuation conductivity as well as the derivation of the results presented in Sec. II. The formalism we use is a generalization of the approach introduced in Ref. 21 so as to include pair-breaking effects. We outline the main steps of the derivation in a condensed form in order to make the paper self-contained. In Sec. IV, we specialize to the parallel magnetic field case and compare our results to those obtained in Ref. 12 using the traditional diagrammatic approach. The results of the comparison are summarized in Table I. Section V is devoted to films in a tilted magnetic field. Here, we present the derivation of the results presented in Sec. II B. Eventually, in Sec. VI we conclude. Some technical details of the calculation are relegated to two Appendixes.

\section{RESULTS}

In this section, we present the main results of our study. We split the discussion into two parts. In the first part, we present the results for the general theory of fluctuation transport in superconductors subject to a magnetic field. In the second part, we discuss the fluctuation conductivity of a thin disordered film in a tilted magnetic field. The presentation is intended to be self-contained. Details of the derivation are described in Secs. III and V.

\section{A. General results: Disordered superconductors in a magnetic field}

The main result of this paper are expressions for the fluctuation conductivity in disordered superconductors subject to a magnetic field. In Sec. II B, we discuss the case of a thin film in a tilted magnetic field as an application. The general results, however, are applicable not only to thin films, but also to several other geometries, for example, wires, cylindric tubes, and nanoribbons. The difference between these examples lies in the spectrum of superconducting fluctuations. In the following, we present the equation determining the fluctuation spectrum [Eq. (1)], and write the general results 
for the corrections to conductivity [Eqs. (3) to (9)]. Then, we briefly discuss the range of applicability.

\section{Fluctuation spectrum}

The fluctuation propagator of the superconducting order parameter field [cf. Eqs. (10) and (11)] is diagonal in the basis of eigenfunctions determined by the following eigenvalue equation:

$$
-\frac{D}{2}[\nabla-2 i e \mathbf{A}(\mathbf{r})]^{2} \phi_{n}(\mathbf{r})=\alpha_{n} \phi_{n}(\mathbf{r}) .
$$

This equation is similar to the single-particle Schrödinger equation in quantum mechanics. Here, however, it is related to the motion of Cooper pairs. Due to the diffusive nature of cooperons, the mass entering the conventional Schrödinger equation is replaced by the inverse of the diffusion constant $1 / D$. The solutions of this equation depend on the external magnetic field and on the geometry of the system, as the equation needs to be supplemented with appropriate boundary conditions. For the boundary to an insulator or vacuum, the following condition should be chosen:

$$
\mathbf{n} \cdot(\nabla-2 i e \mathbf{A}) \phi=0,
$$

where $\mathbf{n}$ is the vector normal to the boundary. This condition corresponds to the requirement of zero supercurrent through the boundary.

The information about the eigenfunctions $\phi_{n}$ and corresponding eigenvalues $\alpha_{n}$ for a certain geometry is sufficient in order to obtain the fluctuation corrections.

\section{General expressions for fluctuation corrections}

We now present the results for the fluctuation corrections (in this section and the rest of this paper, we set $\hbar=1$ ). We write the total correction as the sum of three parts

$$
\delta \sigma=\delta \sigma_{\mathrm{DOS}}+\delta \sigma_{\mathrm{an}}+\delta \sigma_{\mathrm{sc}}
$$

corresponding to the classification in the Usadel equation scheme. The density of states contribution takes the form

$$
\delta \sigma_{\mathrm{DOS}}=2 D e^{2} \int \frac{d \omega}{2 \pi} \sum_{n} \rho_{n n}\left[\mathcal{B}^{\prime} \operatorname{Re} \mathcal{E}_{n}^{\prime} \operatorname{Im} L_{n}-\mathcal{B} \operatorname{Im}\left(\mathcal{E}_{n}^{\prime \prime} L_{n}\right)\right] .
$$

This correction originates from the suppression of the quasiparticle density of states near the Fermi surface.

The anomalous Maki-Thompson correction reads as

$$
\delta \sigma_{\mathrm{an}}=2 D e^{2} \int \frac{d \omega}{2 \pi} \sum_{n} \rho_{n n} \frac{\mathcal{B}^{\prime}}{\alpha_{n}} \operatorname{Im} L_{n} \operatorname{Im} \mathcal{E}_{n} .
$$

This correction may be interpreted as a resonantly enhanced interference effect in the Cooper channel.

The correction induced by the fluctuating supercurrent is conveniently written as the sum of three terms

$$
\delta \sigma_{\mathrm{sc}}=\delta \sigma_{\mathrm{SC}}^{(1)}+\delta \sigma_{\mathrm{SC}}^{(2 a)}+\delta \sigma_{\mathrm{SC}}^{(2 b)},
$$

where

$\delta \sigma_{\mathrm{sc}}^{(1)}=-D e^{2} \int \frac{d \omega}{2 \pi} \sum_{n m} \frac{\boldsymbol{d}_{n m} \mathcal{B}}{\alpha_{n}-\alpha_{m}}\left(\mathcal{E}_{n}^{\prime}-\mathcal{E}_{m}^{\prime}\right)\left(L_{n}-L_{m}\right)$ and

$$
\begin{gathered}
\delta \sigma_{\mathrm{sc}}^{(2 a)}=-D e^{2} \int \frac{d \omega}{2 \pi} \sum_{n m} \frac{\boldsymbol{d}_{n m} \mathcal{B}^{\prime}}{\alpha_{n}-\alpha_{m}} \operatorname{Im}\left(\mathcal{E}_{n}-\mathcal{E}_{m}\right) \operatorname{Im}\left(L_{n}-L_{m}\right), \\
\delta \sigma_{\mathrm{sc}}^{(2 b)}= \\
D e^{2} \int \frac{d \omega}{2 \pi} \sum_{n m} \frac{\boldsymbol{d}_{n m} \mathcal{B}^{\prime}}{\alpha_{n}-\alpha_{m}} \operatorname{Re}\left(\mathcal{E}_{n}-\mathcal{E}_{m}\right) \\
\times \operatorname{Re}\left[L_{n}-L_{m}+\left(\mathcal{E}_{n}^{*}-\mathcal{E}_{m}\right) L_{n} L_{m}^{*}\right] .
\end{gathered}
$$

We introduced the retarded fluctuation propagator in equilibrium

$$
L_{n}(\omega)=\frac{1}{\mathcal{E}_{n}(\omega)}
$$

where

$$
\mathcal{E}_{n}(\omega)=\ln \frac{T_{c 0}}{T}+\psi\left(\frac{1}{2}\right)-\psi\left(\frac{1}{2}+\frac{2 \alpha_{n}-i \omega}{4 \pi T}\right),
$$

and $\psi$ denotes the digamma function. ${ }^{35} \mathcal{B}(\omega)=\operatorname{coth}(\omega / 2 T)$ is the bosonic equilibrium distribution function. The prime in the above set of formulas denotes a derivative with respect to frequency $f^{\prime}(\omega)=\partial_{\omega} f(\omega)$. We further introduced the following matrix elements in the basis of eigenfunctions:

$$
\begin{gathered}
\rho_{n m}(\mathbf{r})=\phi_{n}^{*}(\mathbf{r}) \phi_{m}(\mathbf{r}), \\
\boldsymbol{d}_{n m}(\mathbf{r})=\frac{1}{2} \hat{\mathbf{E}} \cdot \mathbf{r}_{n m}\left(\phi_{n}(\mathbf{r})[\nabla+2 i e \mathbf{A}(\mathbf{r})] \phi_{m}^{*}(\mathbf{r})\right. \\
\left.-\phi_{m}^{*}(\mathbf{r})[\nabla-2 i e \mathbf{A}(\mathbf{r})] \phi_{n}(\mathbf{r})\right),
\end{gathered}
$$

where $\hat{\mathbf{E}}$ is the unit vector in the direction of the external electric field and we further defined

$$
\mathbf{r}_{n m}=\int d \mathbf{r} \mathbf{r} \phi_{n}^{*}(\mathbf{r}) \phi_{m}(\mathbf{r}) .
$$

From the form of the expressions for $\delta \sigma_{\mathrm{sc}}$ [Eqs. (7) to (9)] and using the relation $\boldsymbol{d}_{n m}=-\boldsymbol{d}_{m n}^{*}$, it follows that only the real part of $\boldsymbol{d}_{n m}$ contributes, which turns out to be sufficient for the longitudinal conductivity we study here. When considering the transversal conductivity, where $\boldsymbol{d}_{n m}$ is purely imaginary, a particle-hole symmetry-breaking term needs to be added to $L$ for a nonzero result, and the formulas given above do not hold. $^{21}$

We note that the index $n$, used in general to enumerate the eigensystem of Eq. (1), might in fact be a multi-index with several components. It is also possible that $n$ does not enumerate a discrete set, but rather a continuum. In that case, the sum over $n$ has to be replaced by the corresponding integral.

An important remark is in order here. The anomalous MakiThompson correction diverges in the absence of a magnetic field, as then $\alpha_{0} \rightarrow 0$. The correction may be regularized by introducing a finite dephasing rate $1 / \tau_{\phi} \cdot{ }^{1}$ Dephasing can be provided by magnetic impurities, electron-electron, or electron-phonon collisions. For low temperatures, electronelectron collisions dominate. Outside the region of strong fluctuations, one can consider the dephasing rate as energy independent and equal to the sum of rates due to the Coulomb ${ }^{36}$ and Cooper channels. ${ }^{37,38}$ In our study, we will treat $1 / \tau_{\phi}$ as a phenomenological parameter; it may be introduced into the theory by replacing $\alpha_{n} \rightarrow \alpha_{n}+1 / 2 \tau_{\phi}$ in the formulas for $\delta \sigma$ and $\mathcal{E}_{n}$ given above. 
In principle, the equations presented above can be used to obtain the fluctuation corrections to conductivity for any sample along an unconfined direction by solving the eigenvalue problem (1) for the given geometry. In the next section, we briefly list a number of cases, for which these formulas can be applied.

\section{Eigenvalues $\alpha_{n}$ for different geometries}

In a bulk sample, in the absence of a magnetic field, the eigenvalue equation (1) can be solved by Fourier transformation due to translational invariance, resulting in the following eigenfunctions and eigenvalues:

$$
\phi_{q}(\mathbf{r})=e^{i \mathbf{q} \cdot \mathbf{r}}, \quad \alpha_{q}=\frac{1}{2} D q^{2} .
$$

Films and nanowires have a reduced dimensionality. For the unconfined directions, it is useful to introduce a continuous Fourier transformation, while in the transverse direction(s), modes are quantized. This remains true if a parallel field is applied because it is possible to introduce a vector potential that depends only on the transverse coordinate(s). The eigenvalues

$$
\alpha_{q n}=\frac{1}{2} D q^{2}+\alpha_{\perp n}
$$

can be written as a sum of a continuous and a discrete component.

If the transverse direction is small in extent compared to the superconducting coherence length $\xi_{0}$, often only the lowest transverse mode is relevant. The lowest eigenvalue $\alpha_{\perp 0}$ then plays the role of a pair-breaking parameter. ${ }^{2,12}$ For example, for a film in a parallel magnetic field one finds

$$
\alpha_{\perp 0}=\frac{1}{6} D(e B)^{2} d^{2},
$$

where $d$ is the thickness of the film.

For a film with a perpendicular field, the situation is different from the one discussed above since the vector potential A can not be chosen to be translationally invariant within the plane. The eigenvalues are degenerate Landau levels

$$
\alpha_{n}=2 e D B\left(n+\frac{1}{2}\right) \text {, }
$$

which should be supplemented with suitably chosen eigenfunctions. ${ }^{39}$ For this case, the fluctuation conductivity has been discussed with the help of the Usadel equation approach in Ref. 21.

In this paper, we show that in a tilted field with both perpendicular and parallel field components, the relevant eigenvalues $\alpha_{n}$ can be written as a sum of the eigenvalues for the perpendicular and parallel magnetic field cases. We will discuss the fluctuation corrections for this case in detail.

Another interesting quasi-one-dimensional system is a cylindric shell, i.e., a nanowire with annular cross section. ${ }^{3,8}$ Here, a parallel magnetic field also gives rise to a flux threading the cylinder. The dependence of the lowest eigenvalue $\alpha_{\perp 0}$ on the flux is then (in the limit of vanishing thickness) periodic with the superconducting flux quantum $\varphi_{0}^{*}=h / 2 e$, as can be seen from solving the eigenproblem (1). This special case will be discussed in a separate publication. ${ }^{40}$

\section{B. Film in a tilted magnetic field}

We will now discuss in detail the fluctuation corrections for a thin film in a tilted magnetic field. We consider a thin

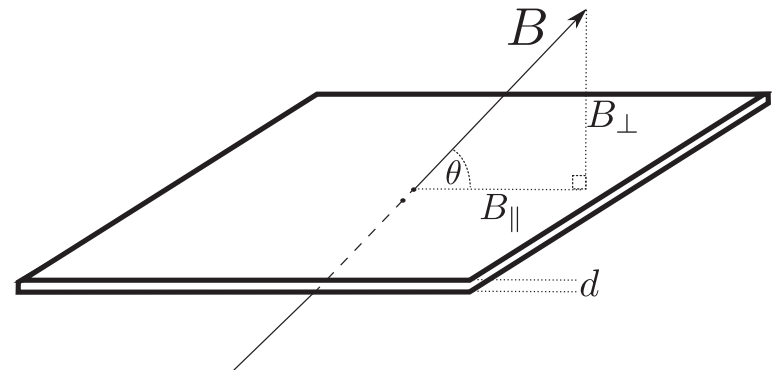

FIG. 1. A sketch of the system under study. A superconducting film of thickness $d$ is penetrated by a magnetic field of magnitude $B$ at an angle $\theta$.

amorphous superconducting film of thickness $d$ penetrated by a magnetic field at an angle $\theta, 0 \leqslant \theta \leqslant \pi / 2$, measured between the field lines and the sample. We study the dirty limit, i.e., $T_{c 0} \tau \ll 1$, where $T_{c 0}$ is the critical temperature of the superconductor and $\tau$ is the elastic scattering time of the electrons. We choose coordinates so that the film lies in the $x-y$ plane, and the magnetic field $\mathbf{B}$ can be written as (see Fig. 1)

$$
\mathbf{B}=B \sin \theta \hat{z}+B \cos \theta \hat{\boldsymbol{y}} .
$$

Here, $\hat{z}$ and $\hat{\boldsymbol{y}}$ are unit vectors in the $z$ and $y$ directions, respectively. We will sometimes use the notation $B_{\|}=B \cos (\theta)$ and $B_{\perp}=B \sin (\theta)$.

The film is assumed to be sufficiently thin so that the condition $d \ll \xi_{0}$ is fulfilled, where $\xi_{0} \approx 0.36 \sqrt{D / T_{c 0}}$ is the superconducting coherence length at zero temperature. ${ }^{1}$ In this limit, the film can be considered as two dimensional as far as its superconducting properties are concerned, whereas the electron motion is assumed to be three dimensional. In the following, we neglect the destructive effect on superconductivity caused by the direct coupling of the magnetic field to the magnetic moment of the electrons. It is known that this is a good approximation for perpendicular magnetic fields and weakly disordered films $\epsilon_{F} \tau \gg 1$, where $\epsilon_{F}$ is the Fermi energy. ${ }^{1}$ For parallel magnetic fields, there is a minimum thickness $d_{\text {Clog }}=\xi_{0} / \epsilon_{F} \tau$ below which paramagnetic effects start to dominate. This is known as the Clogston limit, ${ }^{41,42}$ and we will assume that it is not reached, i.e., that $d \gg d_{\text {Clog }}$. We note that recently the fluctuation conductivity in the opposite paramagnetic limit has also been addressed theoretically. ${ }^{20}$

\section{Phase diagram}

The experimental phase diagram of the film is three dimensional. It is spanned by the temperature and the magnetic field, which is further characterized by its magnitude $B$ and direction $\theta$. A cut of the mean field phase diagram for zero temperature is displayed in Fig. 2. The phase boundary of the superconducting film is determined by the pair-breaking parameter $\alpha$, which quantifies the effectiveness of the magnetic field to suppress superconductivity. ${ }^{2}$ In the considered case of a tilted magnetic field, the pair-breaking parameter is the sum of the contributions due to the parallel and perpendicular field components $\alpha=\alpha_{\|}+\alpha_{\perp}$, where

$$
\begin{aligned}
\alpha_{\perp} & =D e B \sin \theta, \\
\alpha_{\|} & =\frac{D d^{2}}{6}(e B \cos \theta)^{2} .
\end{aligned}
$$




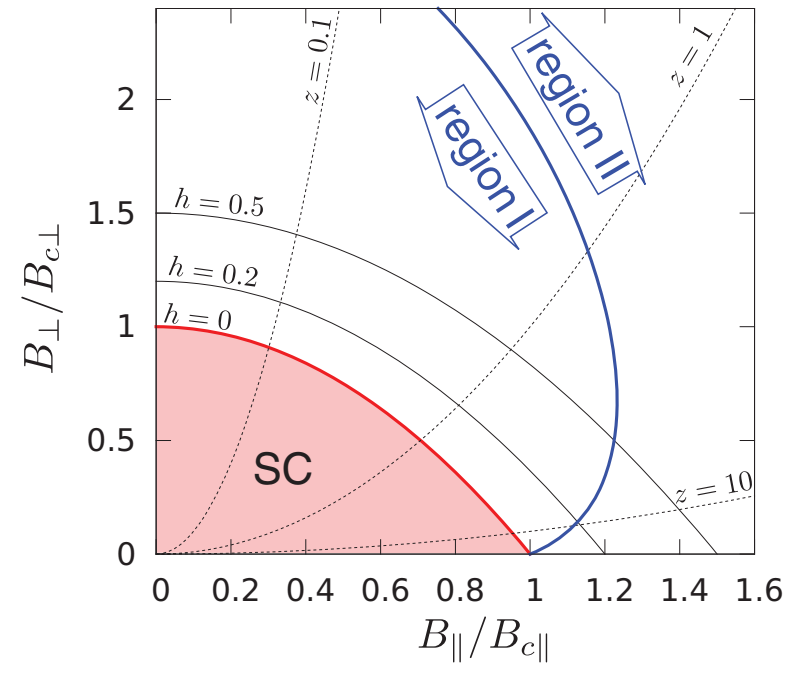

FIG. 2. (Color online) Phase diagram of the film in a tilted magnetic field at zero temperature. The diagram is parametrized by parameters $h$ and $z$, illustrated by lines of constant $h$ (solid line) and constant $z$ (dashed line). SC denotes the region of superconductivity. Regions I and II refer to the asymptotic regions in which we evaluate the corrections to conductivity. They are separated by the line $h=2 /(1+z)$. The sharp angular dependence of the negative correction to conductivity discussed below is a result of the pinching of region II near $B_{c \|}$.

Here, $D=\frac{1}{3} v_{F}^{2} \tau$ is the electronic diffusion constant of the material, where $v_{F}$ is the Fermi velocity.

The critical pair-breaking parameter $\alpha_{c}$, which separates the normal phase with $\alpha>\alpha_{c}$ from the superconducting phase with $\alpha<\alpha_{c}$, is temperature dependent. It is implicitly defined by the equation

$$
\ln \left(\frac{T}{T_{c 0}}\right)-\psi\left(\frac{1}{2}\right)+\psi\left(\frac{1}{2}+\frac{\alpha_{c}(T)}{2 \pi T}\right)=0,
$$

where $\psi(x)$ is the digamma function. ${ }^{35}$ This equation has no solution for temperatures $T>T_{c 0}$, for which the system is a normal metal. By applying the asympotic expansion $\psi(x) \approx$ $\ln x$ for large $x$, one finds that for zero temperature $\alpha_{c 0}=\frac{\pi T_{c 0}}{2 \gamma}$, where $\ln \gamma \approx 0.577$ is the Euler constant.

Once the solution $\alpha_{c}(T)$ is known for arbitrary temperatures, the phase boundary can be constructed. It is a twodimensional surface in the three-dimensional phase diagram spanned by the perpendicular and parallel components of the magnetic field $B_{\perp}$ and $B_{\|}$and by temperature. The phase boundary is determined by the equation

$$
\alpha_{\perp}+\alpha_{\|}=\alpha_{c}(T) .
$$

In order to find the critical field as a function of temperature at a fixed angle $\theta$, for example, one should insert the expressions for $\alpha_{\perp}$ and $\alpha_{\|}$of Eq. (20) into Eq. (22) and obtain a quadratic equation for the critical field strength $B_{c}(\theta, T)$. For a cut at constant temperature $T \leqslant T_{c 0}$, it is convenient to present Eq. (20) in the form ${ }^{43,44}$

$$
\left(\frac{B_{c}(\theta, T) \cos \theta}{B_{c \|}(T)}\right)^{2}+\frac{B_{c}(\theta, T) \sin \theta}{B_{c \perp}(T)}=1,
$$

where $B_{c \perp}$ and $B_{c \|}$ are the (temperature-dependent) critical fields for $\theta=\pi / 2$ and 0 , respectively. They can be obtained by setting $\alpha_{\|, \perp}=\alpha_{c}(T)$ and resolving for $B$. Equation (23) describes a parabolic phase boundary in the $\left(B_{\perp}-B_{\|}\right)$plane.

So far, the effect of a finite dephasing time was not included. As pointed out before, it may be accounted for by a shift in the eigenvalues $\alpha_{n} \rightarrow \alpha_{n}+1 / 2 \tau_{\phi}$. Therefore, the condition for the mean-field transition can be written as $\alpha=\tilde{\alpha}_{c}(T)$, where $\tilde{\alpha}_{c}(T)=\alpha_{c}(T)-1 / 2 \tau_{\phi}$ is modified due to the presence of dephasing effects. If the dephasing time is weakly magnetic field dependent, then its main effect on Eq. (23) is to renormalize the critical fields $B_{c \|}$ and $B_{c \perp}$.

We note that $B_{c \perp}(T=0)$ coincides with the nucleation critical field $B_{c 2}$. For low temperatures $T \rightarrow 0$, the two critical fields $B_{c \|}$ and $B_{c \perp}$ are related by

$$
\frac{B_{c \|}(T=0)}{B_{c \perp}(T=0)}=\frac{1}{d} \sqrt{\frac{6 D}{\alpha_{c 0}}}=4.16 \times \frac{\xi_{0}}{d},
$$

where $\xi_{0}$ is the zero-temperature coherence length. ${ }^{1}$

\section{Parametrization for the vicinity of the quantum critical line}

When formulating the results for the film in a tilted magnetic field below, we will specifically discuss the vicinity of the quantum critical line in the phase diagram, i.e., we concentrate on low temperatures $t=T / T_{c 0} \ll 1$. For the fluctuation conductivity, the regime of small temperatures is particularly interesting. This regime displays the phenomenon of the nonmonotonic magnetoresistance (NM), as was first shown for the perpendicular magnetic field in Ref. 11 and for the parallel magnetic field in Ref. 12. Here, we will discuss this phenomenon for magnetic fields tilted at arbitrary angles $\theta$.

For a fixed temperature, the phase boundary has the shape of a parabola [cf. Eq. (23)]. In order to present the results for the fluctuation corrections, we introduce a parametrization of the region close to this critical line, which will be described in the following.

First, choose an arbitrary angle $\theta$ between $0^{\circ}$ and $90^{\circ}$. Equations (21) and (23) determine the critical field strength $B_{c}(\theta)$, at which, for a given angle, the system undergoes the phase transition. The strength of the magnetic field can then be measured by the relative distance $h$ to the phase boundary:

$$
h=\frac{B-B_{c}(\theta)}{B_{c}(\theta)},
$$

with $h=0$ corresponding to a point on the critical line and $h>0$ corresponding to a point in the normal region of the phase diagram.

In order to parametrize the angle $\theta$, it turns out to be useful to introduce another dimensionless number $z$, which is defined as the ratio between the two projected pair-breaking parameters

$$
z(B, \theta)=\frac{\alpha_{\|}}{\alpha_{\perp}}=\frac{e B d^{2}}{6} \frac{\cos ^{2} \theta}{\sin \theta} .
$$

As can be seen from the definition, $z$ is directly related to the angle, with $z=0$ corresponding to perpendicular field and $z=\infty$ corresponding to parallel field. The parameters $h$ and $z$ can be used instead of $B$ and $\theta$ in order to define a point in the phase diagram. In fact, one can consider $h$ and $z$ as a 
new curvilinear coordinate system of the phase diagram that is aligned along the phase boundary of the system, as depicted in Fig. 2.

Whenever the vicinity of the transition line in the $\left(B_{\perp}-B_{\|}\right)$ plane for a fixed temperature $T \ll T_{c 0}$ is considered, the $B$ dependence of $z$ may be neglected and one may approximate $z \approx z\left[B=B_{c}(\theta, T), \theta\right]$. This quantity can be determined experimentally (without explicit reference to the thickness $d$ ):

$$
z\left[B=B_{c}(\theta, T), \theta\right]=\frac{B_{c \perp}(T)}{B_{c}(\theta, T) \sin \theta}-1 .
$$

For almost parallel magnetic field, $\theta \ll 1$, and for $T \rightarrow 0$, one finds

$$
z=\frac{B_{c \perp}(T=0)}{B_{c \|}(T=0)} \frac{1}{\theta}=0.24 \times \frac{d}{\xi_{0}} \frac{1}{\theta}
$$

\section{Results for the fluctuation conductivity}

The kinetic equation approach employed in this paper leads to a rather natural classification of the three distinct contributions to fluctuation conductivity according to the underlying physical mechanisms. We distinguish the density of states contribution $\delta \sigma_{\mathrm{DOS}}$, the anomalous Maki-Thompson term $\delta \sigma_{\mathrm{an}}$, and the contribution of the fluctuating supercurrent $\delta \sigma_{\mathrm{sc}}$, as discussed in the Introduction. It is worth mentioning that this classification differs from the conventional diagrammatic scheme. For the parallel magnetic field case, the precise correspondence between the two formalisms is worked out in Sec. IV, and illustrated in Table I.

We will now state results for the fluctuation conductivity of the film in the vicinity of the quantum critical line, i.e., for $T \ll T_{c 0}(t \ll 1)$. Let us stress again that the general results displayed in Sec. II A cover the entire normal part of the phase diagram (with the exception of the region of strong fluctuations very close to the transition). Here, we focus on the low-temperature regime since it displays the interesting phenomenon of the NM. Whenever possible, we will discuss the origin of the different corrections according to the classification into density of states, anomalous MakiThompson, and supercurrent contributions.

When formulating our results, we make use of the parametrization of the phase diagram in terms of the parameters $h, z$, and $T$ introduced above (see Fig. 2). For the low-temperature regime, it can be expected that the presence of $\tau_{\phi}$ in the fluctuation propagator mainly leads to a shift in the critical line in the $\left(B_{\perp}-B_{\|}\right)$plane. We assume that this shift has already been performed. At the same time, we neglect $\tau_{\phi}$ in the cooperon because at low $T$ and in the vicinity of the critical line, the cooperon is not singular. This is why the presented formulas will not contain any explicit reference to $\tau_{\phi}$.

Comparatively simple semianalytical expressions can be found in two regimes. In region $\mathrm{I}$, defined by the relation $h \ll \frac{2}{1+z}$, Landau-level quantization of the Cooper-pair motion is crucial. Indeed, the dominant contribution to fluctuation conductivity in this regime originates from fluctuations of the lowest Landau level since these fluctuations become singular at the transition. In regime II, for which $h \gg \frac{2}{1+z}$, the spectrum may be approximated by a continuum for the purpose of the calculation. The reason is that in this regime either, for small $z$, the distance to the transition line is comparatively large and the fluctuations of all levels are nonsingular or, for large $z$, the magnetic field is almost parallel and the distance between adjacent Landau levels becomes very small. The two regions are displayed in the diagram of Fig. 2.

With the only exception of very small angles $\theta$, the system is in region I of the phase diagram when approaching the transition line (compare Fig. 2). In turn, for large magnetic fields far from the transition $h \gg 2$, the system is in region II independent of the value of $z$. The crossover angle between the two regimes near criticality can be estimated from Eq. (28): Region II is reached only for very small angles

$$
\theta \ll 0.12 \times \frac{d}{\xi_{0}} h .
$$

As an illustration, for a film of thickness $d=0.3 \xi_{0}$, fairly close to the transition $h=0.1$, the crossover occurs at an angle $\theta$ of about $0.21^{\circ}$

In the following, we will discuss the two regions separately, starting from region I. After stating the results, we will provide a qualitative discussion of the behavior in the two regions.

Region I. As a special example, region I contains the case of a strictly perpendicular magnetic field $z=0$ in the vicinity of the transition. This case has first been treated in Ref. 11 (see also Ref. 21). Our results for region I can be viewed as a generalization of these previous results to nonperpendicular angles.

The general formulas stated in Sec. II A involve an integration over an internal frequency and the summation over Landau-level indices. As was already noticed in Ref. 11, the most singular contribution in the vicinity of the transition stems from the lowest Landau levels (LL) only. Correspondingly, we consider the contributions due to the singular LL and due to the higher Landau levels (HL) separately. It turns out that for the HL a continuum approximation is sufficient. Furthermore, for both LL and HL contributions we perform a separation into a thermal correction (T), which vanishes for $T \rightarrow 0$, and a quantum correction (0), which is temperature independent and thereby persists even in the limit $T \rightarrow 0$. As a result, the corrections to conductivity may be presented in the following form:

$$
\delta \sigma_{\mathrm{I}}=\delta \sigma_{0, \mathrm{LL}}+\delta \sigma_{\mathrm{T}, \mathrm{LL}}+\delta \sigma_{0, \mathrm{HL}} .
$$

Here, the thermal contribution $\delta \sigma_{\mathrm{T}, \mathrm{LL}}$ reads as follows:

$$
\delta \sigma_{\mathrm{T}, \mathrm{LL}}=\frac{e^{2}}{\pi^{2}}\left(\alpha \tilde{I}_{\alpha}(r)+\beta I_{\beta}(r)\right),
$$

where

$$
\begin{gathered}
\tilde{I}_{\alpha}=\ln r-\frac{1}{2 r}-\psi(r), \\
I_{\beta}=r \psi^{\prime}(r)-\frac{1}{2 r}-1,
\end{gathered}
$$

and we have abbreviated $r=\frac{h}{2 \gamma t}$. This contribution is very similar in structure to the result for the perpendicular magnetic field case derived in Ref. 11. It differs mainly in two respects. First, we omitted the term $\ln h$ from $\tilde{I}_{\alpha}$ as it does not vanish as $T \rightarrow 0$ and is therefore part of the quantum contribution $\delta \sigma_{0, \mathrm{LL}}$ to be discussed below [cf. Eq. (37)]. Second, the prefactors $\alpha$ 
and $\beta$ are now $z$ dependent, i.e., they depend on the angle $\theta$ :

$$
\alpha=\frac{1}{3+z}-\frac{1}{1+z}, \quad \beta=\frac{5+2 z}{3+z}+\frac{1}{1+z} .
$$

In the case of a perpendicular magnetic field, the coefficients reduce to the previously derived $\alpha=-\frac{2}{3}$ and $\beta=\frac{8}{3} \cdot{ }^{11,21}$ Interestingly, however, in approaching the transition $h \rightarrow 0$, we find that $\delta \sigma_{\mathrm{T}, \mathrm{LL}} \approx \frac{2 \gamma e^{2}}{\pi^{2}} \frac{t}{h}$, meaning that the $z$ dependence of the general formula drops out in this limit. When approaching the transition at any finite temperature, $\delta \sigma_{\mathrm{T}, \mathrm{LL}}$ eventually becomes the dominant contribution. It then resembles the well-known Aslamazov-Larkin fluctuation correction. ${ }^{11,23}$

The quantum contribution due to the LL reads as

$$
\delta \sigma_{0, \mathrm{LL}}=\frac{e^{2}}{\pi^{2}}\left[\frac{1}{1+z} \mathrm{Li} \frac{1}{1+h}+G\left(h, \frac{2(1+h)}{z+1}\right)\right],
$$

where $\mathrm{Li}$ is the logarithmic integral function. ${ }^{35}$ The first term stems from the density of states correction $\delta \sigma_{\mathrm{DOS}}$, and the second part is due to the supercurrent correction $\delta \sigma_{\mathrm{sc}}$. The function $G(h, a)$ is defined by the integral

$$
G(h, a)=\frac{1}{2} \int_{1+h}^{\infty} d x \frac{a}{x(x+a)}\left(\frac{1}{\ln x}-\frac{1}{\ln x+a}\right) .
$$

For $h \rightarrow 0$, the contribution $\delta \sigma_{0, \mathrm{LL}}$ can be seen to reduce to

$$
\delta \sigma_{0, \mathrm{LL}} \approx-\frac{e^{2}}{\pi^{2}} \alpha \ln \frac{1}{h} \quad(h \rightarrow 0),
$$

which corresponds to the quantum term in the formula found by Galitski and Larkin. ${ }^{11}$

The last contribution in Eq. (30), $\delta \sigma_{0, \mathrm{HL}}$, was omitted in Ref. 11, and is obtained by considering the higher Landau levels. For this term, one may use the continuum approximation for the sum over Landau levels. This formally corresponds to the limit $z \rightarrow 0$, i.e., this term is only very weakly $z$ dependent. In addition, it is not singular when approaching the transition. Formally, the sum over higher Landau levels is very weakly (doubly logarithmically) divergent, so that it becomes necessary to introduce a high-energy cutoff $\Lambda$ and to take into account only modes with $\alpha_{n}<\Lambda$. As our theory is based on the diffusion approximation, the cutoff can be chosen to be of the order of the transport scattering rate $\Lambda \simeq 1 / \tau$.

The quantum correction from higher Landau levels can then be written as the sum of two integrals:

$$
\begin{aligned}
\delta \sigma_{0, \mathrm{HL}}^{(\mathrm{DOS})}= & -\frac{e^{2}}{2 \pi^{2}} \int_{0}^{\infty} d y \int_{0}^{K} d x \\
& \times \frac{1}{(a+x+y)^{2} \ln (a+x+y)}, \\
\delta \sigma_{0, \mathrm{HL}}^{(\mathrm{sc} 1)}= & \frac{e^{2}}{2 \pi^{2}} \int_{0}^{\infty} d y \int_{0}^{K} d x \\
& \times \frac{x}{(a+x+y)^{3} \ln ^{2}(a+x+y)},
\end{aligned}
$$

where we abbreviated $a=(1+h)^{2}$, and $K$ is a dimensionless cutoff given by $K=\frac{\Lambda}{2 \alpha_{c 0}}$. These integrals can be explicitly solved in terms of the logarithmic integral Li [see Eq. (86) in Sec. V].

As a final remark concerning the correction in region $I$, the thermal contribution originating from higher Landau levels $\delta \sigma_{\mathrm{T}, \mathrm{HL}}$ has been omitted from formula (30) as it is regular and small.

Region II. We now turn to the discussion of region II, where Landau levels are so close that they can entirely be treated in the continuum approximation. In that case, the special significance of the lowest Landau level is lost. As mentioned earlier, the limit of taking a continuous spectrum corresponds to the limit $\theta \rightarrow 0$. The results for a film in a strictly parallel field have been found previously in the diagrammatic technique, ${ }^{12}$ and we agree with these results. For a more detailed discussion of the comparison, we refer to Sec. IV.

We separate the total correction to conductivity in region II into thermal and quantum contributions:

$$
\delta \sigma_{\mathrm{II}}=\delta \sigma_{\mathrm{T}, \mathrm{HL}}+\delta \sigma_{0, \mathrm{HL}} .
$$

The expression for the quantum contribution $\delta \sigma_{0, \mathrm{HL}}$ is the same in both regimes I and II and has already been stated above in Eq. (40). The thermal part is dominated only by the supercurrent correction $\delta \sigma_{\mathrm{sc}}^{(2 b)}$ in the classification introduced in Sec. II A (in the conventional classification, it originates from the Aslamazov-Larkin diagram). It can be written in the form ${ }^{12}$

$$
\delta \sigma_{\mathrm{T}, \mathrm{HL}}=\frac{4 e^{2}}{\pi} F(\eta), \quad \eta=\frac{\alpha-\alpha_{c}(T)}{T} .
$$

For sufficiently small $h$, we can approximate $\eta=2 \pi r$. The function $F$ in the previous formula is defined by

$$
F(\eta)=\frac{\eta}{4 \pi^{2}} \int_{\eta / \pi}^{\infty} \frac{\psi^{\prime}(x) d x}{x}-\frac{1}{4 \pi}-\frac{1}{16 \eta} .
$$

It may be evaluated numerically, and the asymptotic expansion for large and small values of the argument gives

$$
F(\eta)=\left\{\begin{array}{lll}
\frac{\pi}{72 \eta^{2}} & \text { for } & \eta \gg 1 \\
\frac{1}{16 \eta} & \text { for } & \eta \ll 1
\end{array}\right.
$$

We now turn to a more qualitative discussion.

\section{Qualitative discussion: Film in a tilted magnetic field}

Region I. The two dominant corrections in region I are $\delta \sigma_{\mathrm{T}, \mathrm{LL}}$ and $\delta \sigma_{0, \mathrm{LL}}$. They are of different sign when approaching the transition. For small $h \ll t$, in the so-called thermal regime, $\delta \sigma_{\mathrm{T}, \mathrm{LL}}$ dominates. All the corrections $\delta \sigma_{\mathrm{DOS}}, \delta \sigma_{\mathrm{an}}$, and $\delta \sigma_{\mathrm{sc}}$ contribute to $\delta \sigma_{\mathrm{T}, \mathrm{LL}}$ and the net result is a positive correction to conductivity as naively expected when approaching the superconducting state. However, the presence of the term $\delta \sigma_{0, \mathrm{LL}}$, which is negative and dominates in the so-called quantum regime $h \gg t$, leads to a local minimum in $\delta \sigma$ as a function of $h$ at the crossover between the thermal and the quantum regimes. The result is the NM. ${ }^{11,30}$ The physical origin is the negative density of states correction, which competes with the positive supercurrent correction in the quantum regime $h \gg t$, but is numerically larger. The anomalous Maki-Thompson term is ineffective in this regime. The physical mechanism underlying the phenomenon of the NM in region I can therefore be expressed in simple terms. Cooper pairs form, but are comparatively immobile as a consequence of their quantized spectrum (Landau-level quantization). The decrease in conductivity due to a reduction of the density of states of quasiparticles may therefore 


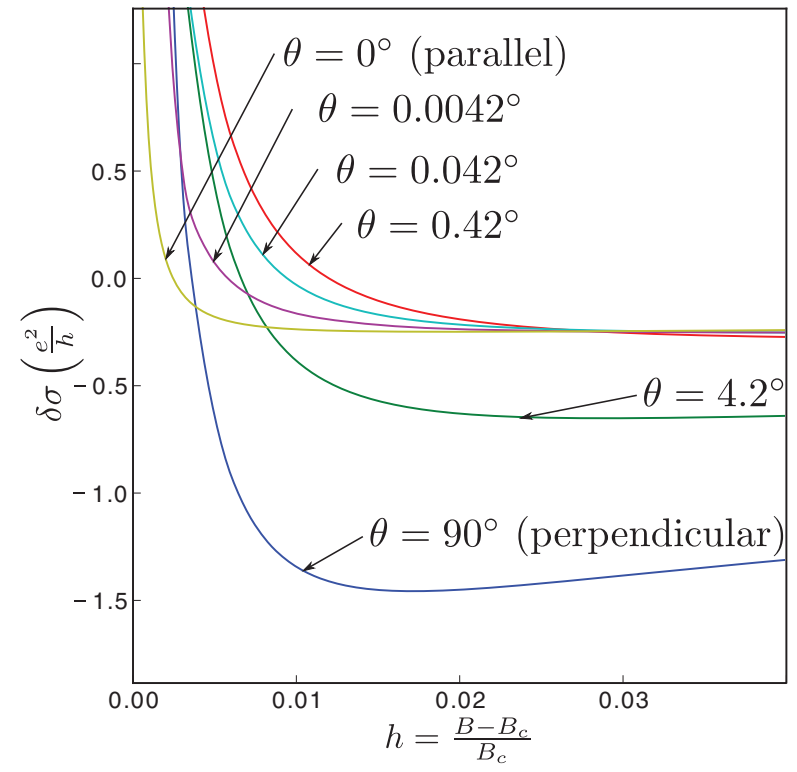

FIG. 3. (Color online) The correction to conductivity of a disordered film at very low temperatures $\left(T=0.005 T_{c 0}, d / \xi_{0}=0.3\right)$. The transition is approached by tuning the magnitude of the magnetic field at a fixed angle $\theta$. When one tilts the angle starting from the perpendicular magnetic field case, the dominant effect is a reduction of the negative correction to conductivity. When tilting further towards parallel fields, a second effect sets in, namely, a sudden decrease of the thermal contribution. This effect becomes visible mainly for small $h$.

overcome the increase in conductivity caused by the fluctuation supercurrent carried by these Cooper pairs.

Crossover between regions I and II. The importance of the term $\delta \sigma_{0, \mathrm{HL}}$ lies in the fact that, irrespective of the precise choice of the cutoff, it is negative and weakly angular dependent. Deep in region I, the phenomenon of the NM is largely determined by the interplay of $\delta \sigma_{0, \mathrm{LL}}$ and $\delta \sigma_{T, \mathrm{LL}}$ as discussed above, and $\delta \sigma_{0, \mathrm{HL}}$ is of minor importance. As one approaches the crossover regime between regions I and II at small angles, however, the negative correction due to $\delta \sigma_{0, \mathrm{LL}}$ diminishes, as it is proportional to $\alpha$, and $\delta \sigma_{0, \mathrm{HL}}$ becomes more relevant. As will be discussed in more detail below, in region II the NM still exists only due to the existence of the negative contribution $\delta \sigma_{0, \mathrm{HL}}$. For the accurate description of the crossover regime itself, a numerical evaluation is necessary and results are displayed in Fig. 3. Let us remind at this point that the negative correction $\delta \sigma_{0, \mathrm{HL}}$ stems from the density of states and supercurrent corrections $\delta \sigma_{\mathrm{DOS}}$ and $\delta \sigma_{\mathrm{sc}}$. Again, the negative density of states contribution dominates.

Another interesting observation can be made in the thermal regime $h \ll t$, which can be reached in both regions by approaching the transition. It is characteristic for this regime that the divergent thermal contributions $\delta \sigma_{\mathrm{T}, \mathrm{LL}}$ and $\delta \sigma_{\mathrm{T}, \mathrm{HL}}$ dominate. As mentioned before, very close to the transition, the system is in region I for almost all angles. The crossover to region II only happens very close to $\theta=0$. It is thus interesting to note that for $h \ll t$, the asymptotic expansion of $\delta \sigma_{\mathrm{T}, \mathrm{LL}}$ contributing to $\delta \sigma_{\mathrm{I}}$ is $\frac{2 \gamma e^{2}}{\pi^{2}} \frac{t}{h}$, while the asymptotic expansion of $\delta \sigma_{\mathrm{T}, \mathrm{HL}}$, which contributes to $\delta \sigma_{\mathrm{II}}$, is $\frac{\gamma e^{2}}{4 \pi^{2}} \frac{t}{h}$. Thus, while crossing over from region I to region II in the thermal regime, the fluctuation correction drops to about $\frac{1}{8}$ of its value for small $h$.

Region II. As mentioned above, $\delta \sigma_{0, \mathrm{HL}}$ is regular at the transition, and slowly decreases when moving towards the normal regime. On the other hand, the thermal contribution $\delta \sigma_{\mathrm{T}, \mathrm{HL}}$ diverges when approaching the transition. As demonstrated in Ref. 12, the interplay of these two contributions also results in a NM. From the preceding discussion it is clear that the nonmonotonic behavior of the magnetoresistance has a different origin for parallel and perpendicular magnetic fields. For parallel fields, the negative correction comes from $\delta \sigma_{0, \mathrm{HL}}$. For perpendicular fields, it originates from $\delta \sigma_{0, \mathrm{LL}}$. In both cases, however, these negative corrections stem from a competition of density of states and supercurrent terms, for which the negative $\delta \sigma_{\text {DOS }}$ dominates over the positive $\delta \sigma_{\mathrm{sc}}$.

This concludes the discussion of the main results. In the following sections, details of the derivation will be presented.

\section{GENERAL FORMALISM}

In this section, we describe the formalism underlying the results presented in Sec. II A. We derive a quasiclassical kinetic equation, the so-called Usadel equation. The form of this equation is slightly nonstandard in order to allow for the inclusion of fluctuations outside the superconducting regime. The formalism used here was introduced in Ref. 21 for a film in a perpendicular magnetic field. We generalize it here in order to be able to treat parallel field components as well. The main difference to Ref. 21 is that a more general set of eigenfunctions and eigenvalues is considered. Otherwise, the derivation is analogous to the one presented in Ref. 21. We describe the main steps here in order to coin the notation and to make the paper self-contained. Previous related works include Ref. 28, in which the Usadel equation was used for the calculation of fluctuation corrections close to $T_{c 0}$ and Refs. 45 and 22, where the Keldysh nonlinear sigma model was employed for the calculation and again, only the vicinity of $T_{c 0}$ was studied. The latter two works are based on the Keldysh sigma model approach for superconductors presented in Ref. 46; the connection between the different formalisms is that the Usadel equation is the saddle-point equation of the sigma model.

\section{A. Microscopic model}

We start from the Keldysh action for electrons with BCS short-range interaction. The interaction in the Cooper channel is already decoupled by means of a Hubbard-Stratonovich transformation, leading to the action

$$
\begin{aligned}
S[\chi, \Delta]= & \int d \mathbf{r} \int_{\mathcal{C}} d t \sum_{\alpha=\uparrow, \downarrow} \chi_{\alpha}^{*}\left(i \partial_{t}-h+\mu\right) \chi_{\alpha} \\
& +\Delta^{*} \chi_{\downarrow} \chi_{\uparrow}+\Delta \chi_{\uparrow}^{*} \chi_{\downarrow}^{*}+\frac{v}{\lambda} \Delta^{*} \Delta .
\end{aligned}
$$

Here, $\chi_{\uparrow}(x)$ and $\chi_{\downarrow}(x)$ are Grassmann fields describing the spin-up and spin-down components of the electrons at the space-time point $x=(\mathbf{r}, t), h$ is the single-particle Hamiltonian, $\Delta(x)$ is the superconducting order parameter field, $v$ is the density of states of electrons per spin direction at 
the Fermi surface, and $\lambda>0$ is a dimensionless parameter, which determines the strength of electron-electron attraction in the Cooper channel. The integral in time is along the closed Keldysh contour $\mathcal{C}{ }^{47}$ The single-particle Hamiltonian

$$
h=-\frac{1}{2 m}[\nabla-i e \mathbf{A}(\mathbf{r})]^{2}+U(\mathbf{r})+e \varphi(\mathbf{r})
$$

includes coupling to a vector potential $\mathbf{A}$ and an external scalar potential $\varphi$, as well as an impurity potential $U$. In this work, we assume a constant potential gradient $\varphi(\mathbf{r})=-\mathbf{E} \cdot \mathbf{r}$, where $\mathbf{E}$ is the electric field.

We separate fields on the forward and backward branches of the Keldysh contour. We further introduce the Nambu $(N)$ particle-hole space, and perform the Keldysh rotation. This means that we describe our system by the fourcomponent fields $\Psi=\left(\psi_{1}, \psi_{2}\right)_{K}^{T}, \psi_{i}=\left(\chi_{\uparrow i}, \chi_{\downarrow i}^{*}\right)_{N}^{T}$ and $\Psi^{\dagger}=$ $\left(\psi_{1}^{\dagger}, \psi_{2}^{\dagger}\right)_{K}, \psi_{i}^{\dagger}=\left(\chi_{\uparrow i}^{*},-\chi_{\downarrow i}\right)_{N}$, where $K$ labels the Keldysh space. This transformation turns the action into

$$
S[\Psi, \check{\Delta}]=\int d x \Psi^{\dagger}\left(i \hat{\tau}_{3} \partial_{t}-\check{H}+\mu+\check{\Delta}\right) \Psi-\frac{2 \nu}{\lambda} \operatorname{tr}\left(\check{\Delta}^{\dagger} \sigma_{1} \check{\Delta}\right) \text {. }
$$

Here and in the following, $\hat{\tau}_{i}$ denote Pauli matrices in particlehole space, while $\hat{\sigma}_{i}$ denote Pauli matrices in Keldysh space. The trace is taken in the four-dimensional product space. Matrices in this product space are denoted as $\check{\Delta}, \check{H}$, etc. The time integration covers the real axis. The single-particle Hamiltontian $\breve{H}$ is obtained from the Hamiltonian $h$ by replacing $\mathbf{A}$ by $\mathbf{A} \hat{\tau}_{3}$. The $4 \times 4$ matrix $\check{\Delta}$ contains the classical (c) and quantum $(q)$ components of the order parameter field $\Delta$ as $\check{\Delta}=\hat{\Delta}_{0} \hat{\sigma}_{0}+\hat{\Delta}_{1} \hat{\sigma}_{1}$, where $\hat{\Delta}_{i}=\Delta_{i} \hat{\tau}_{+}-\Delta_{i}^{*} \hat{\tau}_{-}$, $\hat{\tau}_{ \pm}=\left(\hat{\tau}_{x} \pm i \hat{\tau}_{y}\right) / 2$ and $\vec{\Delta}=\left(\Delta_{c}, \Delta_{q}\right)^{T} \cdot{ }^{21,47}$

In order to perform the quasiclassical approximation, it is useful to define the electronic Green's function

$$
i \check{G}_{\Delta}\left(x, x^{\prime}\right)=\frac{\int[d \Psi] \Psi(x) \Psi^{\dagger}\left(x^{\prime}\right) e^{i S[\Psi, \check{\Delta}]}}{\int[d \Psi] e^{i S[\Psi, \check{\Delta}]}},
$$

where the average is performed over the electronic degrees of freedom only, keeping the field $\breve{\Delta}$ fixed. In order to recover the full Green's function $\breve{G}$, one needs to average as $\breve{G}=$ $\int[d \check{\Delta}] \check{G}_{\Delta} e^{i S_{\mathrm{GL}}[\check{\Delta}]}$ with respect to the Ginzburg-Landau action $S_{\mathrm{GL}}[\check{\Delta}]=-i \ln \int[d \Psi] e^{i S[\Psi, \breve{\Delta}]}$. In Keldysh space, the matrix Green's function has the typical triangular form

$$
\check{G}=\left(\begin{array}{cc}
G^{R} & G^{K} \\
0 & G^{A}
\end{array}\right) .
$$

It is important to note that in general $G_{\Delta}$ does not have the same structure. If $\Delta_{q} \neq 0$, the element in the lower left corner, sometimes referred to as $G_{\Delta}^{Z}$, is not equal to zero. For the linear response calculation of the fluctuation conductivity in the Gaussian approximation, however, it turns out to be sufficient to put $G_{\Delta}^{Z}=0$ as it is proportional to higher powers of the order parameter field. This has been shown in Ref. 21. We will therefore work with the triangular Keldysh structure for $G_{\Delta}$.

In the presence of impurities, the Green's function $\breve{G}$ needs to be averaged over an ensemble of disorder configurations. Physical quantities can be calculated with the help of the disorder averaged Green's function $\left\langle\check{G}_{\Delta}\right\rangle_{\text {dis. }}$. For films with a dimensionless conductance $g \gg 1$, which we consider, it is legitimate to average as $\langle\check{G}\rangle_{\mathrm{dis}}=\int[d \check{\Delta}]\left\langle\check{G}_{\Delta}\right\rangle_{\mathrm{dis}} e^{i\left\langle S_{\mathrm{GL}}[\check{\Delta}]\right\rangle_{\mathrm{dis}}}$, i.e., separately for $G_{\Delta}$ and $S_{\mathrm{GL}}$. Corrections originating from cross correlations between the two terms would be smaller by a factor $1 / g$ than the quantum corrections that are the subject of this paper. The quasiclassical approximation may now be introduced for the disorder averaged Green's function $\left\langle G_{\Delta}\right\rangle_{\text {dis }}$ at a fixed order parameter configuration.

\section{B. Quasiclassical approximation and the Usadel equation}

We assume that $\epsilon_{F}$ is the largest energy scale in the problem, meaning that superconductivity, scattering, and external fields only affect the system close to the Fermi surface: $\Delta, \tau^{-1}, \omega_{\text {ext }} \ll \epsilon_{F}$. Thus, one may use the so-called quasiclassical approximation. ${ }^{48}$ To this end, one transforms to Wigner coordinates and defines the quasiclassical Green's function by integrating over the distance to the Fermi surface

$$
\check{g}_{\mathbf{n}}\left(\mathbf{r}, t, t^{\prime}\right)=\frac{i}{\pi} \int d \xi_{\mathbf{p}}\left\langle\check{G}_{\Delta}\right\rangle_{\mathrm{dis}}\left(\mathbf{r}, \mathbf{p}, t, t^{\prime}\right) .
$$

Here, $\mathbf{n}$ is a unit vector pointing in the direction of $\mathbf{p}$, and $\xi_{\mathbf{p}}=\frac{p^{2}}{2 m}-\mu$. The quasiclassical Green's function $\check{g}_{\mathbf{n}}$ obeys the Eilenberger equation. ${ }^{49}$

In the limit of dirty superconducitivity $T_{c 0} \tau \ll 1$, frequent scattering washes out the angular dependence of the Green's function, which allows us to work with the simpler function $\check{g}=\int d \mathbf{n} \check{g}_{\mathbf{n}}$. The appropriate equation in this limit is the Usadel equation ${ }^{26,48}$

$$
D \hat{\partial}_{\mathbf{A}}\left(\check{g} \hat{\partial}_{\mathbf{A}} \check{g}\right)-\left\{\hat{\tau}_{3} \partial_{t}, \check{g}\right\}+i[\check{\Delta}-\check{\varphi}, \check{g}]=0 .
$$

Here, $D=v_{F}^{2} \tau / 3$ is the electronic diffusion coefficient for diffusion in three dimensions and $\check{\varphi}$ is the external potential, which is considered as a matrix in Nambu and Keldysh space. We defined the derivative $\hat{\partial}_{\mathbf{A}} \check{g}=\nabla \check{g}-i e\left[\mathbf{A} \hat{\tau}_{3}, \check{g}\right]$. Also note that one should read the appearing anticommutator in an operator sense $\left\{\hat{\tau}_{3} \partial_{t}, \check{g}\right\}\left(t_{1}, t_{2}\right)=\tau_{3} \partial_{t_{1}} g\left(t_{1}, t_{2}\right)-\partial_{t_{2}} g\left(t_{1}, t_{2}\right) \tau_{3}$. In this equation and the following, we consider $\breve{g}, \breve{\Delta}$, and $\check{\varphi}$ as matrices in Keldysh and Nambu space and in time. Thus, a multiplication implies the matrix product of the $4 \times 4$ matrix as well as a convolution in time. The Usadel equation has to be supplemented with the constraint $\breve{g}^{2}=\breve{1}$. The current in the sample can be expressed through $\breve{g}$ as ${ }^{50}$

$$
\mathbf{j}=\frac{e \pi v D}{2} \operatorname{tr}\left[\hat{\tau}_{3} \hat{\sigma}_{1}(\check{g} \hat{\nabla} \check{g})\right]
$$

This procedure is an extension of the usual quasiclassical formalism in the Keldysh technique; here, the formalism is applied without employing the mean-field approximation for the field $\Delta$. This allows us to treat the electronic system in the quasiclassical approximation while fluctuations of the order parameter field $\Delta$ can still be taken into account.

In the rest of this section, we consider the normal side of the transition, where fluctuations of the order parameter field are taken into account in the Gaussian approximation. We therefore linearize the Usadel equation using the solution $\Delta=$ 0 as a starting point. This allows us to obtain an expression for the current as a functional of the field $\Delta$, which can then be averaged using the correlation function for $\Delta$. It is possible 
to obtain the corresponding fluctuation propagator from the quasiclassical Green's function itself.

In analyzing fluctuations around the normal state of the metal, we parametrize the Green's function as in Eq. (48), using

$$
\begin{aligned}
g^{R} & =\left(\begin{array}{cc}
1-f f^{*} / 2 & f \\
f^{*} & -1+f^{*} f / 2
\end{array}\right), \\
g^{A} & =\left(\begin{array}{cc}
-1+\bar{f} \bar{f}^{*} / 2 & -\bar{f} \\
-\bar{f}^{*} & 1-\bar{f}^{*} \bar{f} / 2
\end{array}\right),
\end{aligned}
$$

while for the Keldysh component we set $g^{K}=g^{R} h-h g^{A}$. In these equations $g^{K}, g^{R}, g^{A}, h$, and $f$ are all functions of one spatial and two time coordinates $g^{K}=g^{K}\left(\mathbf{r}, t, t^{\prime}\right)$ etc., and the product implies a convolution in time. The parametrization is consistent with the nonlinear constraint $\breve{g}^{2}=\breve{1}$ up to third order in $f$. The functions $f, f^{*}, \bar{f}, \bar{f}^{*}$ are considered to be independent from each other; in fact, it turns out that $f^{*}$ is the conjugate of $f$ only if $\Delta_{q}=0$. Inserting (52) and (53) into the Usadel equation (50), we obtain a linear equation for $f$,

$$
\mathcal{C}^{-1} f=V,
$$

where

$$
V_{t_{1}, t_{2}}(\mathbf{r})=2 i\left[\Delta_{c}\left(\mathbf{r}, t_{1}\right) \delta_{t_{1}-t_{2}}+h_{e}\left(\mathbf{r}, t_{1}-t_{2}\right) \Delta_{q}\left(\mathbf{r}, t_{2}\right)\right],
$$

the operator $\mathcal{C}^{-1}$ is defined as

$$
\mathcal{C}^{-1}=D \partial_{\mathbf{A}}^{2}-\left(\partial_{t_{1}}-\partial_{t_{2}}\right), \quad \partial_{\mathbf{A}}=\nabla-2 i e \mathbf{A}(\mathbf{r}),
$$

and $h_{e}$ will be introduced in Eq. (57) below. For $f^{*}, \bar{f}, \bar{f}^{*}$, similar equations are obtained, which show that $\bar{f}_{t_{1}, t_{2}}=-f_{t_{2}, t_{1}}$, $f_{t_{1}, t_{2}}^{*}=-\bar{f}_{t_{2}, t_{1}}^{*}$, while in general $f^{*} \neq(f)^{*}$.

From the kinetic equation for $h$, to lowest order in $\Delta$, one finds that the only deviation from the equilibrium distribution function is a shift due to the local potential ${ }^{21}$

$$
\begin{gathered}
h(\mathbf{r}, \epsilon)=\operatorname{diag}\left(h_{e}(\mathbf{r}, \epsilon), h_{h}(\mathbf{r}, \epsilon)\right), \\
h_{e / h}(\mathbf{r}, \epsilon)=\mathcal{H}(\epsilon \mp e \varphi(\mathbf{r})) .
\end{gathered}
$$

Here, $\mathcal{H}(\epsilon)=\tanh (\epsilon / 2 T)$ is the fermionic equilibrium distribution function.

In order to solve the linearized Usadel equation for $f$, we introduce the Fourier transform

$$
f_{t_{1}, t_{2}}=\int_{\epsilon_{1} \epsilon_{2}} f_{\epsilon_{1} \epsilon_{2}} e^{-i \epsilon_{1} t_{1}+i \epsilon_{2} t_{2}},
$$

where $\int_{\epsilon}=\int d \epsilon / 2 \pi$. The same convention is used for the independent variables $f^{*}, \bar{f}, \bar{f}^{*}$, and for $V$. In a second step, we expand $f=\sum_{n} f_{n} \phi_{n}, f^{*}=\sum_{n} f_{n}^{*} \phi_{n}^{*}$, etc., and use that the spatial part of the operator $\mathcal{C}^{-1}$ is diagonal in the basis of eigenfunctions $\phi_{n}$ of Eq. (1). It gives

$$
f_{n ; \epsilon_{1}, \epsilon_{2}}=C_{n ; \epsilon_{1}+\epsilon_{2}} \int d \mathbf{r} \phi_{n}^{*}(\mathbf{r}) V_{\epsilon_{1}, \epsilon_{2}}(\mathbf{r}),
$$

where we introduced the so-called cooperon $C_{n}$ :

$$
C_{n ; \epsilon}^{-1}=i \epsilon-2 \alpha_{n}-1 / \tau_{\phi},
$$

including a phenomenological dephasing rate as discussed in Sec. II A.
We now turn to the fluctuation propagator $\mathcal{L}$, which is directly related to the correlation function of the field $\Delta$ :

$$
\left\langle\Delta_{i}\left(x_{1}\right) \Delta_{j}^{*}\left(x_{2}\right)\right\rangle=\frac{i}{2 \nu} \mathcal{L}_{i j}\left(x_{1}, x_{2}\right) .
$$

The indices $i, j$ label the components of the vector $\vec{\Delta}$ defined in Eq. (46). Conveniently, the fluctuation propagator can be found from the quasiclassical Green's function. ${ }^{21}$ We restrict ourselves to the Gaussian approximation for the fluctuations of the order parameter field, i.e., we find the correlator by approximating $S_{\mathrm{GL}}$ by its expansion to second order in $\Delta$. The resulting term contains the Greens function $\breve{G}_{\Delta}$ evaluated at coinciding space points, and can thus be expressed through the quasiclassical Green's function $\breve{g}$. One finds

$$
\begin{aligned}
\left(\mathcal{L}^{-1}\right)_{i j}\left(\mathbf{r}, \mathbf{r}^{\prime}, t, t^{\prime}\right)= & \pi v \frac{\delta}{\delta \Delta_{j}^{*}\left(\mathbf{r}^{\prime}, t^{\prime}\right)} \operatorname{tr} \hat{\sigma}_{i} \hat{\tau}_{-} \breve{g}(\mathbf{r}, t, t) \\
& -\left(\frac{2 v}{\lambda} \hat{\sigma}_{1}\right)_{i j} \delta\left(\mathbf{r}-\mathbf{r}^{\prime}\right) \delta\left(t-t^{\prime}\right) .
\end{aligned}
$$

For the first term on the right-hand side, the identification $\sigma_{c}=1$ and $\sigma_{q}=\sigma_{1}$ is used. The fluctuation propagator can thus be found from the solution of the Usadel equation. It is convenient to work in the basis of eigenfunctions $\psi_{n}$ of (1) and to present the fluctuation propagator in the form $\mathcal{L}\left(\mathbf{r}, \mathbf{r}^{\prime}\right)=$ $\sum_{n m} \psi_{n}(\mathbf{r}) \mathcal{L}_{n m} \psi_{m}^{*}\left(\mathbf{r}^{\prime}\right)$. To first order in the electric field, one finds the following matrix elements:

$$
\begin{aligned}
2 \nu \mathcal{L}_{n m}^{R}(\omega) & =-i\left\langle\Delta_{c, n}(\omega) \Delta_{q, m}^{*}(\omega)\right\rangle \\
& =L_{n} \delta_{n m}-2 \mathbf{E r}_{n m} \mathcal{E}_{m}^{\prime} L_{n} L_{m}, \\
2 \nu \mathcal{L}_{n m}^{A}(\omega) & =-i\left\langle\Delta_{q, n}(\omega) \Delta_{c, m}^{*}(\omega)\right\rangle \\
& =L_{n}^{*} \delta_{n m}-2 \mathbf{E r}_{n m} \mathcal{E}_{n}^{* \prime} L_{n}^{*} L_{m}^{*},
\end{aligned}
$$

and

$$
\begin{aligned}
2 \nu \mathcal{L}_{n m}^{K}(\omega)= & -i\left\langle\Delta_{c, n}^{*}(\omega) \Delta_{c, m}(\omega)\right\rangle \\
= & \mathcal{B}\left(L_{n}-L_{n}^{*}\right) \delta_{n m} \\
& -\mathcal{B}^{\prime} \mathbf{E r}_{n m}\left(L_{m}^{*}-L_{n}+\mathcal{E}_{m} L_{n} L_{m}^{*}-\mathcal{E}_{n}^{*} L_{n} L_{m}^{*}\right) \\
& -2 \mathcal{B} \mathbf{E r}_{n m}\left(\mathcal{E}_{m}^{\prime} L_{n} L_{m}-\mathcal{E}_{n}^{* \prime} L_{n}^{*} L_{m}^{*}\right) .
\end{aligned}
$$

Furthermore, $\mathbf{r}_{n m}$ are the matrix elements of the position operator given in Eq. (14) and $\mathcal{B}(\omega)=\operatorname{coth}(\omega / 2 T)$ is the bosonic equilibrium distribution function. $L_{n}$ is defined in Eqs. (10) and (11). In Eq. (11), $\alpha_{n}$ should be replaced by $\alpha_{n}+1 / 2 \tau_{\phi}$ in order to account for the finite dephasing rate introduced in Eq. (61).

In the next step, we insert the parametrizations (52) and (53) into formula (51). From the normal-metal solution, for which $\hat{g}^{R}=-\hat{g}^{A}=\hat{\tau}_{3}$, one immediately finds the Drude expression for the current $\mathbf{j}^{(n)}=2 v e^{2} D \mathbf{E}$. Taking into account fluctuations up to second order in $f$, one obtains several terms with distinct structure. They can be grouped as follows: ${ }^{21}$

$$
\begin{gathered}
\mathbf{j}^{(\mathrm{DOS})}=-\frac{e^{2} \pi \nu D \mathbf{E}}{2} \int_{\epsilon \epsilon^{\prime}} \mathcal{H}^{\prime}(\epsilon)\left(f_{\epsilon \epsilon^{\prime}}^{*} f_{\epsilon^{\prime} \epsilon}+f_{\epsilon \epsilon^{\prime}} f_{\epsilon^{\prime} \epsilon}^{*}\right), \\
\mathbf{j}^{(\mathrm{an})}=-e^{2} \pi \nu D \mathbf{E} \int_{\epsilon \epsilon^{\prime}} \mathcal{H}^{\prime}(\epsilon)\left(\bar{f}_{\epsilon \epsilon^{\prime}} f_{\epsilon^{\prime} \epsilon}^{*}\right),
\end{gathered}
$$




$$
\begin{aligned}
\mathbf{j}^{(\mathrm{sc})}= & e \pi v D \int_{\epsilon \epsilon^{\prime}} \mathcal{H}(\epsilon)\left(f_{\epsilon \epsilon^{\prime}} \nabla_{-} f_{\epsilon^{\prime} \epsilon}^{*}+\nabla_{-} f_{\epsilon \epsilon^{\prime}}^{*} f_{\epsilon^{\prime} \epsilon}\right. \\
& \left.-f_{\epsilon \epsilon^{\prime}}^{*} \nabla_{+} f_{\epsilon^{\prime} \epsilon}-\nabla_{+} f_{\epsilon \epsilon^{\prime}} f_{\epsilon^{\prime} \epsilon}^{*}\right) .
\end{aligned}
$$

where we abbreviated $\nabla_{ \pm}=\nabla \mp 2 i e \mathbf{A}$. In the result (69), we dropped terms that contain $\mathcal{H}^{\prime}(\epsilon)$. These terms vanish after averaging over fluctuations. The naming of these three components refers to density of states (DOS), anomalous Maki-Thompson (an), and supercurrent (sc), respectively.

Equations (67), (68), and (69) still need to be averaged over the fluctuating order parameter field. To this end, one needs to express $f, f^{*}$, etc. in terms of $\Delta$ and $\Delta^{*}$ and the cooperon, as exemplified Eq. (60). The averaging is then performed with the help of Eq. (62). The integral over the frequency $\epsilon$ can be performed analytically. This step is straightforward in principle, but tedious, this is why we display some intermediate steps in Appendix B. The final results for the fluctuation corrections are displayed in Eqs. (3) to (9) in Sec. II A.

\section{COMPARISON TO THE DIAGRAMMATIC TECHNIQUE}

In this section, we summarize the most important aspects of a comparison between the results for the fluctuation conductivity obtained in this paper (see Sec. II A) and those obtained with the help of the traditional diagrammatic technique in Ref. 12. The comparison is performed for a certain class of pair-breaking transitions considered in Ref. 12, for which our results are also applicable. As discussed in Sec. II A, one- and two-dimensional systems in a parallel magnetic field can be treated on a similar footing. The relevant eigenfunctions of Eq. (1) are plane waves $\phi_{\mathbf{q}}(\mathbf{r})=e^{i \mathbf{q r}}$, where $\mathbf{q}$ is a one- or two-dimensional wave vector. The corresponding eigenvalues have the form $\alpha_{q}=\frac{1}{2} D q^{2}+\alpha$, where $\alpha$ is the so-called pairbreaking parameter. In Appendix A, it is shown that the two sets of formulas for the fluctuation conductivity, one obtained from the diagrammatic calculation and the other one with the help of the Usadel equation approach, can be transformed into each other by means of algebraic manipulations (the ultraviolet cutoff in two and three dimensions requires a special discussion, see Appendix A). This is not only relevant from the point of view of consistency, but it enables us to find the relation between the classification used in Ref. 21 as well as in this paper, and the traditional classification based on diagrams.

In the diagrammatic technique, one deals with five core diagrams, displayed in the leftmost column of Table I. Most of these have topologically similar partner diagrams, so that in total a standard set of 11 diagrams needs to be considered (see Ref. 1). In these diagrams, full lines represent quasiparticle propagators, while wavy lines represent fluctuation propagators. Disorder is taken into account with the help of the so-called impurity cross technique: ${ }^{27}$ shaded boxes and triangles, for example, symbolize cooperons.

The diagrams with one horizontal fluctuation propagator are referred to as density-of-states (DOS) diagrams, while those with vertical fluctuation propagators are called MakiThompson (MT) diagrams. Both of these diagrams may contain either two (2c) or three cooperons (3c). The final diagram is the so-called Aslamazov-Larkin (AL) diagram. It contains two fluctuation propagators. We would like to stress again that this classification is different from the one used in this paper. The authors of Ref. 12 further distinguish terms for which the frequency integral contains the factor $\operatorname{coth}(\omega / 2 T)$ from those that contain $1 / \sinh ^{2}(\omega / 2 T)$. These are denoted as $c h$ and $s h$, respectively; in our notation, they correspond to terms containing the bosonic distribution function $\mathcal{B}$ or its derivative $\mathcal{B}^{\prime}$. The terms $\delta \sigma_{\mathrm{DOS}, c h}^{(2 c)}, \delta \sigma_{\mathrm{MT}, c h}^{(2 c)}$ and $\delta \sigma_{\mathrm{DOS}, c h}^{(3 c)}, \delta \sigma_{\mathrm{MT}, c h}^{(3 c)}$ only differ in their overall coefficient and in Ref. 12 they are called $A$ and $B$, respectively. This naming scheme is also used here to refer to the terms obtained

TABLE I. A comparison of the expressions for the fluctuation conductivity in a parallel magnetic field obtained from a diagrammatic calculation in Ref. 12 and from the Usadel equation approach in this paper. The left-hand side shows the classes of diagrams evaluated in Ref. 12 together with the corresponding terms given in (A3). The right-hand side shows the expressions derived from the Usadel equation technique as given in (A1). Aligned adjacent cells or blocks of cells are (in total) equal.

\begin{tabular}{|c|c|c|c|}
\hline Class of diagrams & Terms in Ref. $12[\mathrm{cf.} \mathrm{Eq.} \mathrm{(A3)]}$ & After integ. by parts & Terms in Usadel technique [cf. Eq. (A1)] \\
\hline \multirow{2}{*}{$\delta \sigma_{\mathrm{DOS}}^{(s h, 2 c)}-A$} & $\delta \sigma_{\mathrm{DOS}}^{(s h, 2)}-A$ & \\
\hline & $\delta \sigma_{\mathrm{DOS}}^{(s h, 3 c)}+\frac{3}{2} B$ & \multicolumn{1}{|c|}{$\frac{1}{2} A-\frac{1}{2} \delta \sigma_{\mathrm{DOS}}^{(s h, 2 c)}$} & \\
\cline { 2 - 3 } & $\delta \sigma_{\mathrm{AL}}^{(c t h)}$ & $-\delta \sigma_{\mathrm{sc}}^{(1)}-\delta \sigma_{\mathrm{sc}}^{(2 a)}$ & \\
\hline
\end{tabular}


in Ref. 12, and they are displayed in the second column of Table I next to the corresponding diagrams. We now briefly describe the manipulations required to convert the equations stated in Ref. 12 to our results. More details can be found in Appendix A. The first step is to perform the integration over fermionic frequencies $\epsilon$ for all terms originating from the DOS and MT diagrams. The result of this integration can be expressed in terms of the propagator $L$. After this step, the algebraic structure is similar to the corresponding terms in the technique used in this paper and first identifications can be made. Finally, it is necessary to integrate by parts in the radial momentum variable $q$. These manipulations lead from column two to column three in Table I, and rearranging the resulting terms to obtain the results from the Usadel technique leads from column three to column four.

In summary, we were able to show that for a certain class of pair-breaking transitions, there is a one-to-one correspondence between the results obtained from the diagrammatic perturbation theory and those obtained from the Usadel equation approach. The results of this comparison are illustrated in Table I.

\section{FILM IN A TILTED MAGNETIC FIELD}

As an application of the formalism developed above, we calculate the fluctuation conductivity for a thin superconducting film penetrated by a magnetic field at an arbitrary angle. We focus on the low-temperature regime, where one can follow in detail the angular dependence of the resistance peak. The main results are summarized and discussed in Sec. II B. Here, we present details of the calculation.

First, one needs to solve Eq. (1) in order to find the eigenmodes and eigenvalues specific for the sample geometry and the orientation of the magnetic field. With this knowledge, the matrix elements $\rho_{n m}$ and $\mathbf{d}_{n m}$ of Eqs. (12) and (13) can be found. When inserted into the general expressions, Eqs. (3) to (9), one can obtain a set of formulas that would in principle allow us to evaluate the fluctuation corrections everywhere in the metallic phase. We will specialize on temperatures $T \ll$ $T_{c 0}$, however, where one finds comparatively simple results. In order to structure the calculation, it turns out to be useful to distinguish quantum contributions, that are independent of temperature, and persist for $T \rightarrow 0$, and thermal ones, which capture the temperature dependence, but vanish in the limit $T \rightarrow 0$. Two asymptotic regions in the phase diagrams are found where the evaluation of the sum over Landau levels can approximately be performed. As the lowest Landau level is of special importance in certain limits, we evaluate the contributions of the lowest and of the higher Landau levels separately.

\section{A. Eigenvalue equation and matrix elements}

Here, we solve the eigenvalue equation (1) for a thin film in a tilted magnetic film. The tilting angle is denoted as $\theta$ (for an illustration see Fig. 1). We describe the magnetic field by a vector potential in Landau gauge

$$
A=\left(-B_{\perp} y+B_{\|} z, 0,0\right)
$$

where we abbreviated $B_{\perp}=B \sin \theta, B_{\|}=B \cos \theta$. The eigenvalue equation (1) then reads as

$$
-\frac{D}{2}\left[\left(\partial_{x}+2 i e B_{\perp} y-2 i e B_{\|} z\right)^{2}+\partial_{y}^{2}+\partial_{z}^{2}\right] \phi=\alpha \phi .
$$

Under the assumption that the layer is thin, only the lowest transverse mode in the $z$ direction is of interest, for which $\phi$ is almost constant as a function of $z$. We drop $\partial_{z}^{2}$ and integrate over $z$ from $-d / 2$ to $d / 2$ :

$-\frac{D}{2}\left(\left(\partial_{x}-2 i e B_{\perp} y\right)^{2}+\partial_{y}^{2}\right) \phi=\left(\alpha-\frac{D}{6} e^{2} B_{\|}^{2} d^{2}\right) \phi$.

We thus find the eigenfunctions

$$
\phi_{n p}=e^{i p y} \chi_{n}\left(x-\frac{p}{2 e B_{\perp}}\right),
$$

where $\chi_{n}$ are the eigenfunctions of a one-dimensional quantum harmonic oscillator with frequency $2 e D B_{\perp}$ and mass $D^{-1}$, and corresponding eigenvalues

$$
\alpha_{n}=2 e D B_{\perp}\left(n+\frac{1}{2}\right)+\frac{D}{6} e^{2} B_{\|}^{2} d^{2} .
$$

The Landau levels are degenerate, as the eigenvalues do not depend on $p$. The perpendicular component of the magnetic field causes Landau-level quantization, and the parallel field component adds a constant to the pair-breaking parameter that is quadratic in $B_{\|}$. It will prove useful to present the eigenvalues in the form

$$
\alpha_{n}=\alpha_{\perp}(2 n+1+z),
$$

where $z$ is introduced in Eq. (26).

The matrix elements required for the evaluation of the general formulas Eqs. (3) to (9) can be found by using well-known relations for the eigenfunctions of a quantum harmonic oscillator. ${ }^{39}$ As the eigenvalues do not depend on $p$, the integration over this variable can immediately be performed. We find

$$
\begin{gathered}
\int_{p, q} \rho_{n p, m q}=\frac{B_{\perp}}{\pi} \delta_{n m}, \\
\int_{p, q} \mathbf{d}_{n p, m q}^{x}=\frac{E B_{\perp}}{2 \pi}\left(m \delta_{n+1, m}-n \delta_{n, m+1}\right) .
\end{gathered}
$$

For the second matrix element, we only calculated the vector component in the $x$ direction, the direction of the electric field. This is sufficient for the calculation of the longitudinal conductivity.

\section{B. Low-temperature approximation, two asymptotic regimes}

In the low-temperature regime $t \ll 1$, the calculations can be considerably simplified by working with the asymptotic form of the fluctuation propagator. The calculation is further structured by separating quantum and thermal contributions as well as contributions of the lowest and of higher Landau levels.

\section{Low-temperature approximation and separation into thermal and quantum contributions}

For $t \ll 1$, we can use the asymptotic expansion for the digamma function and approximate the inverse fluctuation 
propagator (11) as

$$
\mathcal{E}_{n}=-\ln \left(\frac{\alpha_{n}-\frac{i \omega}{2}}{\alpha_{c}}\right) .
$$

Considering further Eqs. (3) to (9), we notice that terms with an integrand containing $\mathcal{B}^{\prime}(\omega)=\partial_{\omega} \operatorname{coth}(\omega / 2 T)$ only contribute at finite temperatures as the integrand vanishes exponentially as the temperature goes to zero. The terms containing $\mathcal{B}$, found from $\delta \sigma_{\mathrm{DOS}}$ and $\delta \sigma_{\mathrm{sc}}^{(1)}$, contribute at finite temperatures as well as in the zero-temperature limit. For these terms, a useful separation can be achieved by writing

$$
\mathcal{B}(\omega)=[\mathcal{B}(\omega)-\operatorname{sgn}(\omega)]+\operatorname{sgn}(\omega),
$$

where the combination in square brackets vanishes exponentially with temperature. The notion quantum terms will be used for those contributions originating from $\operatorname{sgn}(\omega)$ in this decomposition and they will be denoted as $\delta \sigma_{0}$; they are temperature independent. The remaining terms, which encode the temperature dependence and originate either from $\mathcal{B}^{\prime}$ or from the difference $\mathcal{B}(\omega)-\operatorname{sgn}(\omega)$, will be referred to as thermal terms and denoted as $\delta \sigma_{T}$; they vanish in the zero-temperature limit.

\section{Summation over Landau levels}

The evaluation of the formulas for the fluctuation corrections requires a summation over the Landau-level index $n$ which in general is difficult to treat. However, we note that when approaching the transition, $L_{0}=1 / \mathcal{E}_{0}$ diverges for $\omega=0$ while the other $L_{n}$ remain finite. In order to quantify the importance of the $n=0$ term, we introduce the parameter

$$
\lambda=\frac{L_{0}}{L_{1}}-1=\frac{\ln \left(\frac{3+z}{1+z}\right)}{\ln (1+h)} .
$$

The number $\lambda$ measures the relative importance of the lowest Landau level as compared to higher ones. The equations $\lambda \gg 1$ and $\lambda \ll 1$ define the regions I and II, respectively, as introduced in Sec. II B.

If $\lambda$ is large, the lowest Landau level is the only strongly contributing mode. The divergence close to the critical line is accounted for by the term $n=0$ in the sum, a restriction to only this term is known as the lowest-Landau-level approximation. This approximation has been put forward by Galitski and Larkin $^{11}$ for the perpendicular magnetic field case $z=0$, and allowed them to obtain closed formulas.

If $\lambda$ is small, the significance of the lowest Landau level is lost. To correctly understand the crossover, we separate each contribution in region I into four parts: First, each sum is split into the lowest-Landau-level $(n=0)$ term (LL), and the sum over higher Landau levels $(n>0, \mathrm{HL})$. Furthermore, it is of calculational advantage to perform a separation of each of the resulting terms into the thermal (T) and quantum (0) contributions as explained above.

\section{Evaluation of integrals in region I}

\section{Thermal terms}

Lowest Landau level ( $L L)$. For the evaluation of the thermal part, only frequencies $\omega \simeq T \ll T_{c 0}$ contribute, and thus (78) can be expanded in $\omega$ and $h$. In order to find all relevant contributions, for the DOS and an contributions it is necessary to expand to second order. The result of this expansion can be written as

$$
\delta \sigma_{\mathrm{T}, \mathrm{LL}}=\frac{e^{2}}{\pi^{2}} \sum_{i} \int_{0}^{\infty} d \omega \frac{\alpha_{i}(\mathcal{B}-1) \omega-\beta_{i} \mathcal{B}^{\prime} \omega^{2}}{\left(2 \alpha_{0} h\right)^{2}+\omega^{2}},
$$

where $i$ enumerates different contributions. The prefactors $\alpha_{i}$ and $\beta_{i}$ are $z$ dependent:

$$
\begin{aligned}
\alpha_{\mathrm{DOS}} & =-\frac{1}{1+z}, \quad \beta_{\mathrm{DOS}}=-\frac{1}{1+z}, \\
\alpha_{\mathrm{an}} & =0, \quad \beta_{\mathrm{an}}=\frac{2}{1+z}, \\
\alpha_{\mathrm{sc}}^{(1)} & =\frac{1}{3+z}, \quad \beta_{\mathrm{sc}}^{(1)}=0, \\
\alpha_{\mathrm{sc}}^{(2 a)} & =0, \quad \beta_{\mathrm{sc}}^{(2 a)}=\frac{1}{3+z}, \\
\alpha_{\mathrm{sc}}^{(2 b)} & =0, \quad \beta_{\mathrm{sc}}^{(2 b)}=\frac{4+2 z}{3+z} .
\end{aligned}
$$

The integrals can be evaluated as a sum over poles of $\mathcal{B}$ and $\mathcal{B}^{\prime}$. The result is the expression for $\delta \sigma_{\mathrm{T}, \mathrm{LL}}$ presented in Eq. (31).

As compared to the result of Galitski and Larkin, ${ }^{11}$ we have modified $\tilde{I}_{\alpha}$ by subtracting $\ln (h)$, which is part of the quantum component (it will be treated separately below), and we added the angular dependence via $z$. Care must also be taken as Galitski and Larkin employed the traditional classification based on diagrams, while we use here the classification introduced in Ref. 21.

Higher landau levels (HL). The quantum contribution due to the higher Landau levels are not singular at the transition and can be neglected. When going to smaller angles, the thermal contribution due to higher Landau levels increases. The point where the higher levels start to play a role marks the onset of the crossover between regions I and II.

\section{Quantum terms}

Lowest Landau level ( $L L)$. The quantum part of the lowestLandau-level contribution consists of terms originating from $\delta \sigma_{\mathrm{DOS}}$ and $\delta \sigma_{\mathrm{sc}}^{(1)}$. Contributions to the integral are not restricted to $\omega<T$ now, thus linearization of the integrand is not appropriate here as it is for the thermal part. In these terms, $\omega$ only appears in $\mathcal{E}$, thus we can make a change of variables $i \omega \rightarrow \tilde{\omega}$. The integration contour of $\tilde{\omega}$ can then be rotated by $90^{\circ}$ onto the real axis, thus making the integrand real.

Turning first to the quantum part of the DOS correction (4), it can be written as

$$
\delta \sigma_{0, \mathrm{LL}}^{(\mathrm{DOS})}=-\frac{e^{2}}{2 \pi^{2}}\left(\frac{1+h}{1+z}\right) \int_{1+h}^{\infty} \frac{d x}{x^{2} \ln x},
$$

and the integral can be expressed in terms of the logarithmic integral function ${ }^{35} \mathrm{Li}(x)=\int_{0}^{x} \frac{d z}{\ln z}$, resulting in the first term in Eq. (35). The second contribution to the quantum part originates from $\sigma_{\mathrm{sc}}^{(1)}$ of Eq. (7). Upon setting $a=\frac{2(1+h)}{1+z}$, it can be written as

$$
\delta \sigma_{0, \mathrm{LL}}^{(\mathrm{sc} 1)}=\frac{e^{2}}{2 \pi^{2}} \int_{1+h}^{\infty} d x\left[\frac{1}{x}-\frac{1}{x+a}\right]\left[\frac{1}{\ln x}-\frac{1}{\ln x+a}\right] .
$$


This gives the second term in Eq. (35). In order to approximate this integral, we note that in the limit $h \ll a$, the asymptotic behavior at the phase boundary is due to the singularity of the first inverse logarithm:

$$
\delta \sigma_{0, \mathrm{LL}}^{(\mathrm{sc} 1)}=\frac{e^{2}}{2 \pi^{2}} \frac{a}{a+1} \ln h \quad(h \ll a) .
$$

Furthermore, the next term in this asymptotic expansion around small $h$ is an $a$-dependent constant. For more general values of $h$, the integral needs to be evaluated numerically.

Higher Landau levels (HL). The quantum contributions from $n>0$ are nonsingular at the transition. Still, one needs to be more careful than for the thermal contribution because the sum over all levels is in fact divergent, and a cutoff has to be introduced. We convert the sum over $n$ in the expressions (4) and (7) into an integral. This transformation becomes exact in the limit $\theta \rightarrow 0$, and it is a good approximation otherwise.

The resulting integrals are doubly logarithmically divergent, so a cutoff $\Lambda$ must be introduced, as discussed before Eq. (40). After introducing dimensionless integration variables and again rotating the contour for $\omega$, we arrive at the integrals $\delta \sigma_{0, \mathrm{HL}}^{(\mathrm{DOS})}$ and $\delta \sigma_{0, \mathrm{HL}}^{(\mathrm{scl})}$ given in Eq. (40).

These integrals can also be expressed explicitly in terms of the logarithmic integral $\operatorname{Li}(x)$. The correction then takes the form

$$
\begin{aligned}
\delta \sigma_{0, \mathrm{HL}}= & \frac{e^{2}}{2 \pi^{2}}\left[K \operatorname{Li}\left(\frac{1}{K}\right)+K^{2} \operatorname{Li}\left(\frac{1}{K^{2}}\right)\right. \\
& \left.-\ln \frac{\ln K}{\ln a}+\mathcal{R}\left(a^{2}, K^{2}\right)-2 \mathcal{R}(a, K)\right],
\end{aligned}
$$

where we introduced the function

$$
\mathcal{R}(x, y)=x\left[\operatorname{Li}\left(\frac{1}{x}\right)-\operatorname{Li}\left(\frac{1}{y}\right)\right]
$$

and abbreviated $a=(1+h)^{2}$ and $K=\Lambda /\left(2 \alpha_{c 0}\right)$, and $\Lambda$ is the energy cutoff up to which superconducting fluctuations are taken into account. As discussed in Sec. II B, it is of the order of the elastic scattering rate of electrons $1 / \tau$.

\section{Evaluation of integrals in region II}

For region II, the lowest Landau level loses its special significance. The summation over Landau levels can be replaced by an integration. For the quantum part $\delta \sigma_{0, \mathrm{HL}}$, the result was already given in the previous section in Eq. (86). We thus only need to consider the thermal term $\delta \sigma_{\mathrm{T}, \mathrm{HL}}$ here.

In contrast to region I, one needs to integrate over Landau levels also for the thermal contribution; an expansion of $\mathcal{E}$ in $\omega$ and $h$, however, is again possible. After applying this expansion to all thermal terms, one finds that the term $\delta \sigma_{\mathrm{sc}}^{(2 b)}$ is dominant over all other terms, which are reduced by the factor $T / T_{c 0}$, which in the considered regime is small. This is in contrast to the case of the LL, where all terms contribute with equal magnitude.

The dominant term $\delta \sigma_{\mathrm{sc}}^{(2 b)}$ can then be written as $\frac{4 e^{2}}{\pi} F(\eta)$, with $F(\eta)$ given by an integral over $q$ and $\omega$ (Ref. 12):

$$
F(\eta)=\int \frac{d^{2} k d y}{(2 \pi)^{2}} \frac{1}{\sinh ^{2} y} \frac{k^{2} y^{2}}{\left[\left(\eta+k^{2}\right)^{2}+y^{2}\right]^{2}} .
$$

Here, $\eta$ is given by $\eta=\left[\alpha_{0}(h)-\alpha_{c}\right] / T$, which for low temperatures can be written as $\eta=\frac{\pi}{2 \gamma t}\left[2 h+h^{2}\right]$.

The integration in $y$ can be performed analytically after writing $1 / \sinh ^{2} y$ as a sum of its poles, resulting in the following expression:

$$
\begin{aligned}
F(\eta)= & -\frac{1}{4 \pi^{2}} \int_{\eta / \pi}^{\infty} d x(\pi x-\eta) \\
& \times\left[\frac{1}{x} \psi^{\prime}(x)+\psi^{\prime \prime}(x)+\frac{1}{2 x^{3}}\right] .
\end{aligned}
$$

The integral $F$ can further be simplified to give Eq. (42). The asymptotic behavior of $F$ for large and small values of $\eta$ as stated below Eq. (43) can be found by inserting the asymptotic expansions $\psi^{\prime}(x) \approx x^{-2}$ for small $x$ and $\psi^{\prime}(x)=$ $x^{-1}+x^{-2} / 2+x^{-3} / 6+\cdots$ for large $x$.

\section{CONCLUSION}

We studied the fluctuation conductivity of disordered superconductors subject to a magnetic field in the metallic phase using a quasiclassical kinetic equation approach. The derived expressions generalize the results of Ref. 21, in which calculations were performed for films in perpendicular magnetic fields, to a more general class of pair-breaking transitions in superconductors of different geometries. We were also able to make contact with previously derived formulas for films and wires in parallel fields. ${ }^{12}$ For the parallel magnetic field case we performed a detailed comparison between the traditional classification based on diagrams with the classification based on the Usadel equation approach (see Table I). As an application, we studied fluctuation corrections in a superconducting film subject to a tilted field with emphasis on the low-temperature regime where these films display the phenomenon of the NM. The calculations performed in Ref. 21 and in this paper clearly show in which way different physical mechanisms contribute to this phenomenon: The reduced quasiparticle density of states leads to an increase in resistance, while Cooper pairs do not efficiently transport charge in this regime and are thus unable to compensate this effect.

It should be noted that aside from the fluctuation corrections, additional quantum corrections exist in disordered electronic systems. Both the weak localization correction and the Altshuler-Aronov interaction correction are logarithmically divergent at low temperatures. These corrections do not become singular near the phase transition, nevertheless, they need to be taken into account in a quantitative comparison to experimental data. The dephasing time $\tau_{\phi}$ has been introduced phenomenologically in this paper (see Sec. II A). For vanishing magnetic fields, dephasing is necessary in order to regularize the anomalous Maki-Thompson correction. At low temperatures and finite magnetic fields, no regularization is required, but dephasing may still influence the magnetic field and temperature dependence of the fluctuation corrections. For example, it leads to a change in the phase boundary.

The phenomenon of the NM is of particular relevance in the context of the magnetic field-tuned superconductor-insulator transition observed in thin disordered superconducting films. ${ }^{51}$ In these films, one finds a change from a superconducting to an insulating behavior $(d \rho / d T<0)$ as a function of the magnetic 
field which leads to a very pronounced resistance maximum at low temperatures. For suitably prepared films, the resistance at the maximum may exceed the normal-state resistance by many orders of magnitude. ${ }^{52}$ Unfortunately, transport in the highly resistive phase is very difficult to describe theoretically. The NM predicted ${ }^{11}$ and also observed ${ }^{5,30}$ in low-resistive films may be viewed as a precursor of this effect. Importantly, for low-resistive samples, controlled calculations can be performed, as was done in this paper. One may hope that a comparison to experimental data may contribute to a better understanding of the phenomenon of the NM and of the properties of the thin films in general.

\section{ACKNOWLEDGMENTS}

We are very grateful for discussions with A. Finkel'stein, Ch. Fräßdorf, Y. Oreg, and K. Tikhonov. We thank J. Behrmann for help with the preparation of the manuscript. G.S. acknowledges support by the Alexander von Humboldt Foundation.

\section{APPENDIX A: COMPARISON TO DIAGRAMMATIC PERTURBATION THEORY}

In this appendix, we show how the results of the diagrammatic calculation of Ref. 12 and those obtained in the Usadel equation approach can be transformed into each other. The systems under study are films or wires subject to a parallel magnetic field.

\section{Fluctuation corrections obtained from the Usadel equation technique}

In Ref. 12, the authors studied the fluctuation conductivity of systems for which the fluctuation spectrum is continuous in one or two dimensions and strongly discretized in the transversal direction(s). For these systems, the eigenvalues of Eq. (1) are given by Eq. (16). The authors assume that only the lowest mode $n=0$ is of relevance. The corresponding eigenfunctions are plane waves in the unconfined directions $\psi_{\mathbf{q}}=e^{i \mathbf{q r}}$, where $\mathbf{q}$ is a one- or two-dimensional wave vector.

Under these assumptions, one can also obtain expressions for the fluctuation corrections from the general results presented in Sec. II A. Inserting the matrix elements of Eqs. (12) and (13), restricting the sum over transverse modes to $n=0$ and writing $\alpha_{\perp 0}=\alpha$, the expressions for the fluctuation corrections can be written as

$$
\begin{aligned}
\delta \sigma_{\mathrm{DOS}} & =2 D e^{2} \int \frac{d \omega}{2 \pi} \frac{d^{d} q}{(2 \pi)^{d}}\left[\mathcal{B}^{\prime} \operatorname{Re} \mathcal{E}^{\prime} \operatorname{Im} L-\mathcal{B} \operatorname{Im}\left(\mathcal{E}^{\prime \prime} L\right)\right] \\
\delta \sigma_{\mathrm{an}} & =2 D e^{2} \int \frac{d \omega}{2 \pi} \frac{d^{d} p}{(2 \pi)^{d}} \frac{\mathcal{B}^{\prime}}{D q^{2} / 2+\alpha} \operatorname{Im} L \operatorname{Im} \mathcal{E} \\
\delta \sigma_{\mathrm{sc}}^{(1)} & =4 D^{2} e^{2} \int \frac{d \omega}{2 \pi} \frac{d^{d} q}{(2 \pi)^{d}} q_{x}^{2} \mathcal{B} \operatorname{Re}\left(\mathcal{E}^{\prime \prime} \mathcal{E}^{\prime} L^{2}\right) \\
\delta \sigma_{\mathrm{sc}}^{(2 a)} & =-4 D^{2} e^{2} \int \frac{d \omega}{2 \pi} \frac{d^{d} q}{(2 \pi)^{d}} q_{x}^{2} \mathcal{B}^{\prime} \operatorname{Re} \mathcal{E}^{\prime} \operatorname{Re}\left(L^{2} \mathcal{E}^{\prime}\right) \\
\delta \sigma_{\mathrm{sc}}^{(2 b)} & =16 D^{2} e^{2} \int \frac{d \omega}{2 \pi} \frac{d^{d} q}{(2 \pi)^{d}} q_{x}^{2} \mathcal{B}^{\prime} \operatorname{Im} \mathcal{E}^{\prime} \operatorname{Re}\left(\mathcal{E}^{\prime} L\right) \operatorname{Im} L
\end{aligned}
$$

where $\mathbf{q}$ is the $d$-dimensional wave vector along the unconfined direction(s) ( $d=1$ for wires, $d=2$ for films), we remind that $\mathcal{B}(\omega)=\operatorname{coth}(\omega / 2 T)$ is the bosonic distribution function

$$
\mathcal{E}_{q}(\omega)=\ln \frac{T_{c 0}}{T}+\psi\left[\frac{1}{2}\right]-\psi\left[\frac{1}{2}+\frac{D q^{2}+2 \alpha+i \omega}{4 \pi T}\right],
$$

and $L=1 / \mathcal{E}$ is the fluctuation propagator. In the displayed formulas, the momentum and frequency arguments $(q, \omega)$ have been suppressed for the sake of brevity.

\section{Fluctuation corrections obtained in Ref. 12}

We next compare the results (A1) to the results derived diagrammatically in Ref. 12 . In that publication, the following fluctuation corrections are presented (we adjusted them to the notation used in this paper):

$$
\begin{aligned}
\delta \sigma_{\mathrm{DOS}}^{(s h, 2 c)} & =4 i D e^{2} \int \frac{d^{d} q d \epsilon d \omega}{(2 \pi)^{d} 2 \pi} \mathcal{H}(\epsilon) \mathcal{B}^{\prime}(\omega) L_{q}^{*}(\omega) \operatorname{Re}\left[C_{q}^{2}(2 \epsilon-\omega)\right], \\
\delta \sigma_{\mathrm{DOS}}^{(s h, 3 c)} & =-8 i D e^{2} \int \frac{d^{d} q d \epsilon d \omega}{(2 \pi)^{d} 2 \pi} \mathcal{H}(\epsilon) \mathcal{B}^{\prime}(\omega) L_{q}^{*}(\omega) \operatorname{Re}\left[D q_{x}^{2} C_{q}^{3}(2 \epsilon-\omega)\right], \\
\delta \sigma_{\mathrm{MT}}^{(s h)} & =4 i D^{2} e^{2} \int \frac{d^{d} q d \epsilon d \omega}{(2 \pi)^{d} 2 \pi} \mathcal{H}(\epsilon) \mathcal{B}^{\prime}(\omega) L_{q}^{*}(\omega) C_{q}(2 \epsilon-\omega) C_{q}(-2 \epsilon+\omega), \\
-2 A & =-16 D e^{2} \int \frac{d^{d} q d \epsilon d \omega}{(2 \pi)^{d} 2 \pi} \mathcal{H}(\epsilon) \mathcal{B}(\omega)\left[C_{q}^{3}(2 \epsilon-\omega) L_{q}^{*}(\omega)\right], \\
\frac{9}{2} B & =-72 D e^{2} \int \frac{d^{d} q d \epsilon d \omega}{(2 \pi)^{d} 2 \pi} \mathcal{H}(\epsilon) \mathcal{B}(\omega) L_{q}^{*}(\omega) D q_{x}^{2} C_{q}^{4}(2 \epsilon-\omega), \\
\delta \sigma_{\mathrm{AL}}^{(s h)} & =-8 D e^{2} \int \frac{d^{d} q d \omega}{(2 \pi)^{d} 2 \pi} \mathcal{B}^{\prime}(\omega) D q_{x}^{2}\left[\left\{\operatorname{Re}\left[L_{q}(\omega) \mathcal{E}_{q}^{\prime}(\omega)\right]\right\}^{2}-\operatorname{Im}\left[L_{q}(\omega)\left(\mathcal{E}_{q}^{\prime}(\omega)\right)^{2}\right] \operatorname{Im} L_{q}(\omega)\right], \\
\delta \sigma_{\mathrm{AL}}^{(c t h)} & =16 D e^{2} \int \frac{d^{d} q d \omega}{(2 \pi)^{d} 2 \pi} \mathcal{B}(\omega) D q_{x}^{2} \operatorname{Re}\left[L_{q}^{2}(\omega) \mathcal{E}_{q}^{\prime}(\omega) \mathcal{E}_{q}^{\prime \prime}(\omega)\right] .
\end{aligned}
$$

The corresponding diagrams are shown in Table I. 


\section{Comparison}

The first five terms in Eq. (A3) are not yet integrated with respect to the fermionic frequency $\epsilon$, while the last two terms are already very similar in structure to $\delta \sigma_{\mathrm{sc}}^{(1)}, \delta \sigma_{\mathrm{sc}}^{(2 a)}$, and $\delta \sigma_{\mathrm{sc}}^{(2 b)}$ in (A1). In fact, one can verify that

$$
\begin{gathered}
4 \delta \sigma_{\mathrm{sc}}^{(1)}=\delta \sigma_{\mathrm{AL}}^{(c t h)}, \\
2 \delta \sigma_{\mathrm{sc}}^{(2 a)}+\delta \sigma_{\mathrm{sc}}^{(2 b)}=\delta \sigma_{\mathrm{AL}}^{(s h)} .
\end{gathered}
$$

The integration in $\epsilon$ for the first five terms in Eq. (A3) can be performed analytically. The required manipulations are similar to those described in Appendix B. The results are

$$
\begin{aligned}
\delta \sigma_{\mathrm{DOS}}^{(s h, 2 c)} & =4 e^{2} D \int \frac{d^{d} q d \omega}{(2 \pi)^{d+1}} \mathcal{B}^{\prime} \operatorname{Im} L \operatorname{Re} \mathcal{E}^{\prime}, \\
\delta \sigma_{\mathrm{DOS}}^{(s h, 3 c)} & =-4 e^{2} D \int \frac{d^{d} q d \omega}{(2 \pi)^{d+1}} \mathcal{B}^{\prime} D q_{x}^{2} \operatorname{Im} L \operatorname{Im} \mathcal{E}^{\prime \prime}, \\
-2 A & =-8 e^{2} D \int \frac{d^{d} q d \omega}{(2 \pi)^{d+1}} \mathcal{B} \operatorname{Im}\left[L \mathcal{E}^{\prime \prime}\right], \\
\frac{9}{2} B & =-12 e^{2} D \int \frac{d^{d} q d \omega}{(2 \pi)^{d+1}} \mathcal{B} D q_{x}^{2} \operatorname{Re}\left[L \mathcal{E}^{\prime \prime \prime}\right], \\
\delta \sigma_{\mathrm{MT}}^{(s h)} & =2 e^{2} D \int \frac{d^{d} q d \omega}{(2 \pi)^{d+1}} \frac{1}{\alpha_{q}} \mathcal{B}^{\prime} \operatorname{Im} L \operatorname{Im} \mathcal{E} .
\end{aligned}
$$

Comparing these expressions to (A1), we find

$$
\begin{gathered}
2 \delta \sigma_{\mathrm{DOS}}=\delta \sigma_{\mathrm{DOS}}^{(s h, 2 c)}-A, \\
\delta \sigma_{\mathrm{an}}=\delta \sigma_{\mathrm{MT}}^{(s h)} .
\end{gathered}
$$

Finally, by transforming the momentum integrals to spherical coordinates and integrating by parts in the radial variable, one can show that

$$
\begin{gathered}
\frac{1}{2} \delta \sigma_{\mathrm{sc}}^{(2 a)}=-\delta \sigma_{\mathrm{DOS}}^{(s h, 2 c)}-\delta \sigma_{\mathrm{DOS}}^{(s h, 3 c)}, \\
0=\frac{3}{2} A-3 \delta \sigma_{\mathrm{sc}}^{(1)}-\frac{9}{2} B .
\end{gathered}
$$

We note that for dimensions $d \geqslant 2$, some of the terms in Eqs. (3) to (9) require an ultraviolet regularization. Indeed, an upper cutoff $\frac{1}{2} D q^{2} \leqslant \Lambda$ for the momentum integral needs to be introduced as discussed in connection with Eq. (40). In $2 d$ this divergence is very weak (doubly logarithmic), in $3 d$ it is more severe. This is not unusual as we work with a low-energy theory that ceases to be accurate at higher energies. The important point is that the theory captures correctly the sensitivity to temperature and magnetic fields. It should be remarked in this context that the boundary term, which we dropped when performing the integration by parts to obtain Eqs. (A9) and (A10), is in fact of order $\Lambda^{d-2} / \ln \Lambda$. The boundary term that arises once a finite cutoff is introduced, however, is insensitive to small changes in the parameters $T$ and $h$. We therefore do not attribute particular importance to the difference in the ultraviolet regularization of the terms obtained from the Usadel equation and from the diagrammatic technique.

The identities (A4), (A5), (A7), (A8), (A9), and (A10) show that our results, Eqs. (3) to (9), when applied to the case under study, are equal to the corrections derived diagrammatically [Eq. (A3)] in one spatial dimension. In two and three dimensions, the equivalence still holds up to details of the ultraviolet regularization. The identification of corresponding terms is summarized in Table I.

\section{APPENDIX B: FREQUENCY INTEGRATION}

The derivation of Eqs. (3) to (9) from Eqs. (67), (68), and (69) as well as the derivation of Eq. (A6) from Eq. (A3) both involve an integration over the "fermionic" frequency $\epsilon$. By way of example, we provide some details on the evaluation of the integrals. The integrand typically comprises two cooperon propagators $C_{n}(\epsilon)=\left(i \epsilon-2 \alpha_{n}\right)^{-1}$, multiplied by one or more factors of the fermionic equilibrium distribution function $\mathcal{H}_{\epsilon}=\tanh (\epsilon / 2 T)$ or its derivatives.

Integrals with two cooperons can be treated by a partial fraction decomposition

$$
\begin{aligned}
A_{n m} & =\int_{\epsilon} \mathcal{H}_{\epsilon} C_{n}(2 \epsilon-\omega) C_{m}(2 \epsilon-\omega) \\
& =\frac{1}{2\left(\alpha_{n}-\alpha_{m}\right)} \int_{\epsilon} \mathcal{H}_{\epsilon}\left[C_{n}(2 \epsilon-\omega)-C_{m}(2 \epsilon-\omega)\right] \\
& =\frac{\mathcal{E}_{n}^{*}(\omega)-\mathcal{E}_{m}^{*}(\omega)}{4 i \pi\left(\alpha_{n}-\alpha_{m}\right)}
\end{aligned}
$$

where we used the shorthand notation $\int_{\epsilon}=\int \frac{d \epsilon}{2 \pi}$. The integral has been performed by the method of residues, the poles of $\mathcal{H}_{\epsilon}$ lie on the imaginary axis at $\epsilon_{n}=4 \pi i T(n+1 / 2)$, with $n$ integer. The resulting sum can be expressed in terms of the digamma function, and further in terms of $\mathcal{E}$ [Eq. (11)]. For $\alpha_{m}=\alpha_{n}$, one may use the relation $\partial_{\alpha_{n}} \mathcal{E}_{n}(\omega)=-2 i \partial_{\omega} \mathcal{E}_{n}(\omega)$ and finds

$$
A_{n n}=-\frac{1}{2 \pi} \mathcal{E}_{n}^{* \prime}(\omega)
$$

Integrals involving $\mathcal{H}_{\epsilon-\omega}$ can be reduced to the discussed examples by a shift in the integration variable and complex conjugation, e.g.,

$$
\int_{\epsilon} \mathcal{H}_{\epsilon-\omega} C_{n}^{2}(2 \epsilon-\omega)=-A_{n n}^{*} .
$$

Another useful relation, which can be obtained in a similar way, is

$$
\begin{aligned}
\bar{A}_{n m} & =\int_{\epsilon} \mathcal{H}_{\epsilon} C_{n}(2 \epsilon-\omega) C_{m}(-2 \epsilon+\omega) \\
& =\frac{\mathcal{E}_{n}^{*}(\omega)-\mathcal{E}_{m}(\omega)}{4 i \pi\left(\alpha_{n}+\alpha_{m}\right)} .
\end{aligned}
$$

Finally, integrals including $\mathcal{H}_{\epsilon}^{\prime}$ can by reduced to the former integrals using integration by parts, yielding

$$
\begin{aligned}
B_{n m} & =\int_{\epsilon} \mathcal{H}_{\epsilon}^{\prime} C_{n}(2 \epsilon-\omega) C_{m}(2 \epsilon-\omega)=2 A_{n m}^{\prime}, \\
\bar{B}_{n m} & =\int_{\epsilon} \mathcal{H}_{\epsilon}^{\prime} C_{n}(2 \epsilon-\omega) C_{m}(-2 \epsilon+\omega)=2 \bar{A}_{n m}^{\prime} .
\end{aligned}
$$

If the integrand contains more than one factor of $\mathcal{H}_{\epsilon}$ or $\mathcal{H}_{\epsilon}^{\prime}$, the following two identities can be used to simplify the expression:

$$
\begin{gathered}
\mathcal{H}_{\epsilon} \mathcal{H}_{\epsilon-\omega}=1-\mathcal{B}_{\omega}\left(\mathcal{H}_{\epsilon}-\mathcal{H}_{\epsilon-\omega}\right), \\
\mathcal{H}_{\epsilon}^{\prime} \mathcal{H}_{\epsilon-\omega}=-\mathcal{B}_{\omega} \mathcal{H}_{\epsilon}^{\prime}-\mathcal{B}_{\omega}^{\prime}\left(\mathcal{H}_{\epsilon}-\mathcal{H}_{\epsilon-\omega}\right) .
\end{gathered}
$$


The first identity is essentially the addition theorem for tanh, ${ }^{35}$ while the second identity is obtained from the first one in three steps: A shift $\epsilon \rightarrow \epsilon+\omega$ is followed by a differentiation with respect to $\omega$ and a second shift $\epsilon \rightarrow \epsilon-\omega$.

With the stated integration formulas at hand, in combination with relations (B7) and (B8), all the required integrals can be performed. Let us give an example, which is a step required for obtaining Eq. (4) from Eq. (67):

$$
\begin{aligned}
\int_{\epsilon} & \mathcal{H}_{\epsilon}^{\prime} \mathcal{H}_{\epsilon-\omega} C_{n}^{2}(2 \epsilon-\omega) \\
= & -\int_{\epsilon} C_{n}^{2}(2 \epsilon-\omega)\left[\mathcal{H}_{\epsilon}^{\prime} \mathcal{B}_{\omega}+\mathcal{B}_{\omega}^{\prime} \mathcal{H}_{\epsilon}-\mathcal{B}_{\omega}^{\prime} \mathcal{H}_{\epsilon-\omega}\right] \\
& =-\mathcal{B}_{\omega} B_{n n}-\mathcal{B}_{\omega}^{\prime}\left(A_{n n}+A_{n n}^{*}\right)
\end{aligned}
$$

*tarasinski@lorentz.leidenuniv.nl

${ }^{1}$ A. I. Larkin and A. A. Varlamov, Theory of Fluctuations in Superconductors (Oxford University Press, Oxford, 2004).

${ }^{2} \mathrm{~K}$. Maki, in Superconductivity, edited by R. D. Parks (Marcel Dekker, New York, 1969), p. 1035.

${ }^{3}$ Y. Liu, Y. Zadorozhny, M. M. Rosario, B. Y. Rock, P. T. Carrigan, and H. Wang, Science 294, 2332 (2001).

${ }^{4}$ A. Pourret, H. Aubin, J. Lesueur, C. A. Marrache-Kikuchi, L. Berge, L. Dumoulin, and K. Behnia, Nat. Phys. 2, 683 (2006).

${ }^{5}$ M. Steiner and A. Kapitulnik, Phys. C (Amsterdam) 422, 16 (2005).

${ }^{6}$ N. C. Koshnick, H. Bluhm, M. E. Huber, and K. A. Moler, Science 318, 1440 (2007).

${ }^{7}$ B. Sacepe, C. Chapelier, T. I. Baturina, V. M. Vinokur, M. R. Baklanov, and M. Sanquer, Nat. Commun. 1, 140 (2007).

${ }^{8}$ I. Sternfeld, E. Levy, M. Eshkol, A. Tsukernik, M. Karpovski, H. Shtrikman, A. Kretinin, and A. Palevski, Phys. Rev. Lett. 107, 037001 (2011).

${ }^{9}$ T. I. Baturina, S. V. Postolova, A. Y. Mironov, A. Glatz, M. R. Baklanov, and V. M. Vinokur, Europhys. Lett. 97, 17012 (2012).

${ }^{10}$ N. P. Breznay, K. Michaeli, K. S. Tikhonov, A. M. Finkel'stein, M. Tendulkar, and A. Kapitulnik, Phys. Rev. B 86, 014514 (2012).

${ }^{11}$ V. M. Galitski and A. I. Larkin, Phys. Rev. B 63, 174506 (2001).

${ }^{12}$ A. V. Lopatin, N. Shah, and V. M. Vinokur, Phys. Rev. Lett. 94, 037003 (2005); N. Shah and A. Lopatin, Phys. Rev. B 76, 094511 (2007).

${ }^{13}$ G. Schwiete and Y. Oreg, Phys. Rev. Lett. 103, 037001 (2009).

${ }^{14}$ G. Schwiete and Y. Oreg, Phys. Rev. B 82, 214514 (2010).

${ }^{15}$ A. D. Maestro, B. Rosenow, and S. Sachdev, Ann. Phys. (NY) 324, 523 (2009).

${ }^{16}$ K. Michaeli and A. M. Finkel'stein, Phys. Rev. B 80, 214516 (2009).

${ }^{17}$ M. N. Serbyn, M. A. Skvortsov, A. A. Varlamov, and V. Galitski, Phys. Rev. Lett. 102, 067001 (2009).

${ }^{18}$ A. Glatz, A. A. Varlamov, and V. M. Vinokur, Europhys. Lett. 94, 47005 (2011).

${ }^{19}$ A. Glatz, A. A. Varlamov, and V. M. Vinokur, Phys. Rev. B 84, 104510 (2011).

${ }^{20}$ M. Khodas, A. Levchenko, and G. Catelani, Phys. Rev. Lett. 108, 257004 (2012).

${ }^{21}$ K. S. Tikhonov, G. Schwiete, and A. M. Finkel'stein, Phys. Rev. B 85, 174527 (2012).

${ }^{22}$ A. Petkovic and V. M. Vinokur, arXiv:1301.7298.

${ }^{23}$ L. G. Aslamazov and A. I. Larkin, Fiz. Tverd. Tela 10, 1104 (1968)

[Sov. Phys.-Solid State 10, 875 (1968)].

${ }^{24}$ K. Maki, Prog. Theor. Phys. 39, 897 (1968).

${ }^{25}$ R. S. Thompson, Phys. Rev. B 1, 327 (1970).

${ }^{26}$ K. D. Usadel, Phys. Rev. Lett. 25, 507 (1970).
${ }^{27}$ A. A. Abrikosov, L. P. Gorkov, and I. E. Dzyaloshinski, Methods of Quantum Field Theory in Statistical Physics (Prentice-Hall, New Jersey, 1963).

${ }^{28}$ A. F. Volkov, K. E. Nagaev, and R. Seviour, Phys. Rev. B 57, 5450 (1998).

${ }^{29}$ V. Gantmakher, S. Ermolov, G. Tsydynzhapov, A. Zhukov, and T. Baturina, Pis'ma Zh. Eksp. Teor. Fiz. 77, 498 (2003) [JETP Lett. 77, 424 (2003)].

${ }^{30}$ T. I. Baturina, J. Bentner, C. Strunk, M. R. Baklanov, and A. Satta, Phys. B (Amsterdam) 359, 500 (2005).

${ }^{31}$ V. Gantmakher, M. Golubkov, V. Dolgopolov, A. Shashkin, and G. Tsydynzhapov, Pis'ma Zh. Eksp. Teor. Fiz. 71, 693 (2000) [JETP Lett. 71, 473 (2000)].

${ }^{32}$ K. A. Parendo, L. M. Hernandez, A. Bhattacharya, and A. M. Goldman, Phys. Rev. B 70, 212510 (2004).

${ }^{33}$ A. Johansson, I. Shammass, N. Stander, E. Peled, G. Sambandamurthy, and D. Shahar, Solid State Commun. 151, 743 (2011).

${ }^{34}$ B. L. Altshuler, A. Varlamov, and M. Y. Reizer, Zh. Eksp. Teor. Fiz. 84, 2280 (1983) [Sov. Phys.-JETP 57, 1329 (1983)].

${ }^{35} \mathrm{M}$. Abramowitz and I. Stegun, Handbook of Mathematical Functions (Dover, New York, 1972).

${ }^{36}$ B. L. Altshuler, A. G. Aronov, D. E. Khmelnitskii, and A. I. Larkin, Coherent Effect in Disordered Conductors (MIR, Moscow, 1982).

${ }^{37}$ A. I. Larkin and Y. N. Ovchinnikov, Zh. Eksp. Teor. Fiz. 61, 1221 (1971) [JETP Lett. 34, 651 (1972)].

${ }^{38}$ W. Brenig, M.-c. Chang, E. Abrahams, and P. Wölfle, Phys. Rev. B 31, 7001 (1985).

${ }^{39}$ C. Cohen-Tannoudji, B. Die, and F. Laloe, Quantum Mechanics (Wiley, New York, 1977).

${ }^{40}$ B. Tarasinski and G. Schwiete (unpublished).

${ }^{41}$ A. M. Clogston, Phys. Rev. Lett. 9, 266 (1962).

${ }^{42}$ B. S. Chandrasekhar, Appl. Phys. Lett. 1, 7 (1962).

${ }^{43}$ F. E. Harper and M. Tinkham, Phys. Rev. 172, 441 (1968).

${ }^{44}$ S. I. Vedeneev and Y. N. Ovchinnikov, JETP Lett. 75, 195 (2002) [Pis'ma Zh. Eksp. Teor. Fiz. 75, 228 (2002)].

${ }^{45}$ A. Levchenko and A. Kamenev, Phys. Rev. B 76, 094518 (2007).

${ }^{46}$ M. V. Feigel'man, A. I. Larkin, and M. A. Skvortsov, Phys. Rev. B 61, 12361 (2000).

${ }^{47}$ A. Kamenev, Non-Equilibrium Systems (Cambridge University Press, Cambridge, 2011).

${ }^{48}$ N. Kopnin, Theory of Nonequilibrium Superconductivity (Oxford University Press, New York, 2001).

${ }^{49}$ G. Eilenberger, Z. Phys. B 214, 195 (1968).

${ }^{50}$ J. Rammer and H. Smith, Rev. Mod. Phys. 58, 323 (1986).

${ }^{51}$ A. F. Hebard and M. A. Paalanen, Phys. Rev. Lett. 65, 927 (1990).

${ }^{52}$ G. Sambandamurthy, L. W. Engel, A. Johansson, and D. Shahar, Phys. Rev. Lett. 92, 107005 (2004). 